\title{
Cyclic behaviour and resistance of saturated sand under non-symmetrical loading conditions
}

\author{
J. YANG* and H. Y. SZE*
}

\begin{abstract}
This paper reports findings from an experimental study that aimed to investigate the undrained behaviour of sand in non-symmetrical cyclic loading, and to clarify the role of initial static shear in liquefaction resistance. The testing programme, conducted on a standard sand under triaxial conditions, covers a broad range of initial states in terms of relative density, confining stress and initial shear stress ratio $(\alpha)$. Three distinct failure modes have been identified from the tests: flow-type failure, cyclic mobility and plastic strain accumulation. Of these, flowtype failure, characterised by abrupt runaway deformations without any prior warning, is the most critical, and pertains to sand in the loose state. The tests also demonstrate that the presence of initial static shear stress is beneficial to the liquefaction resistance of loose sand at low $\alpha$ levels, but it becomes detrimental at high $\alpha$ levels. In this connection the concept of threshold $\alpha$ is proposed, together with the use of a no-stress-reversal line for better characterisation of the effect of initial static shear. Furthermore, in the conceptual framework of critical state soil mechanics, a fairly good linear relationship has been established between the threshold $\alpha$ and the state parameter $\psi$ that collectively accounts for the initial relative density and mean stress level. This relationship suggests that the threshold $\alpha$ decreases with increasing values of $\psi$, or with sand becoming looser than the critical state. It is further proposed that the concept of threshold $\alpha$ also applies to sand at high relative density, as long as the confining stress becomes sufficiently high. This proposal leads to a unified and consistent interpretation of the complicated static shear effect.
\end{abstract}

KEYWORDS: earthquakes; failure; laboratory tests; liquefaction; sands; shear strength
La présente communication fournit les conclusions d'une étude expérimentale, dont le but était d'examiner le comportement non drainé du sable en présence de charges cycliques asymétriques, et de clarifier le rôle du cisaillement statique initial dans la résistance à la liquéfaction. Le programme d'essais, effectué sur un sable standard soumis à des conditions triaxiales, couvre une vaste gamme d'états initiaux sur le plan de la densité relative, de la contrainte de confinement et du taux de contrainte de cisaillement initial $(\alpha)$. Ces essais ont permis d'identifier trois modes de rupture distincts, à savoir la rupture par solifluxion, la mobilité cyclique, et l'accumulation d'allongement plastique. De ces différents modes, la rupture par solifluxion, caractérisée par des déformations brutales sans le moindre signal de précaution, est la plus critique; elle concerne le sable à l'état meuble. Les essais démontrent également que la présence de la contrainte de cisaillement statique initiale est bénéfique pour le résistance à la liquéfaction du sable meuble à des niveaux $\alpha$ bas, mais devient préjudiciable en présence de niveaux $\alpha$ élevés. A cet effet, on propose un concept, celui du $\alpha$ limite, ainsi que l'utilisation d'une ligne sans renversement de tension, afin de mieux caractériser l'effet du cisaillement statique initial. En outre, dans le cadre conceptuel de la mécanique des sols à l'état critique, on a établi un rapport linéaire relativement bon entre le $\alpha$ limite et le paramètre d'état $\psi$, qui expriment collectivement la densité relative initiale et le niveau de contrainte moyenne. Ce rapport semble indiquer que le $\alpha$ limite diminue au fur et à mesure qu'augmente $\psi$, ou lorsque le sable devient plus meuble que l'état critique. Sur le même plan, on propose également que le concept du $\alpha$ limite s'applique également au sable à densité relative élevée, tant que la contrainte de confinement reste suffisamment élevée. Cette proposition permet d'obtenir une interprétation homogène de l'effet compliqué du cisaillement statique.

\section{INTRODUCTION}

As a result of the Niigata and Alaska earthquakes of 1964, soil liquefaction has emerged as an important subject area, creating a great deal of interest among researchers and practising engineers. A fundamental understanding of liquefaction behaviour and the mechanisms involved has been developed over the past four decades, mainly through wellcontrolled laboratory tests (e.g. Seed \& Lee, 1966; Castro, 1975; Toki et al., 1986; Ishihara, 1993; Vaid et al., 2001). The liquefaction resistance of saturated sand is commonly evaluated by undrained cyclic triaxial tests with two-way, symmetrical loading in compression and extension. In the

Manuscript received 20 February 2009; revised manuscript accepted 21 December 2009. Published online ahead of print 20 July 2010. Discussion on this paper closes on 1 June 2011, for further details see p. ii.

* Department of Civil Engineering, The University of Hong Kong, Hong Kong. triaxial test a sand sample is consolidated under hydrostatic conditions and then subjected to a cyclic deviatoric stress, which alters between positive and negative values of the same magnitude (Fig. 1(a)).

The symmetrical loading represents level ground conditions in the free field, where no initial static shear stresses act on the horizontal planes of the elements of soil. In many major projects involving earth dams, embankments or slopes, however, soil elements are subjected to static, driving shear stresses on the horizontal planes before the earthquake loading effect is developed. The sloping ground conditions require tests with initial stress conditions different from those shown in Fig. 1(a). Following the early work of Lee \& Seed (1967), the existence of initial shear stresses has been simulated by several researchers using triaxial tests on anisotropically consolidated samples (e.g. Vaid \& Chern, 1983; Hyodo et al., 1994). In so doing, anisotropic consolidation is conducted under the major and minor principal stresses, $\sigma_{1 \mathrm{c}}^{\prime}$ and $\sigma_{3 \mathrm{c}}^{\prime}$, to give rise to a static shear stress $\tau_{\mathrm{s}}$ 

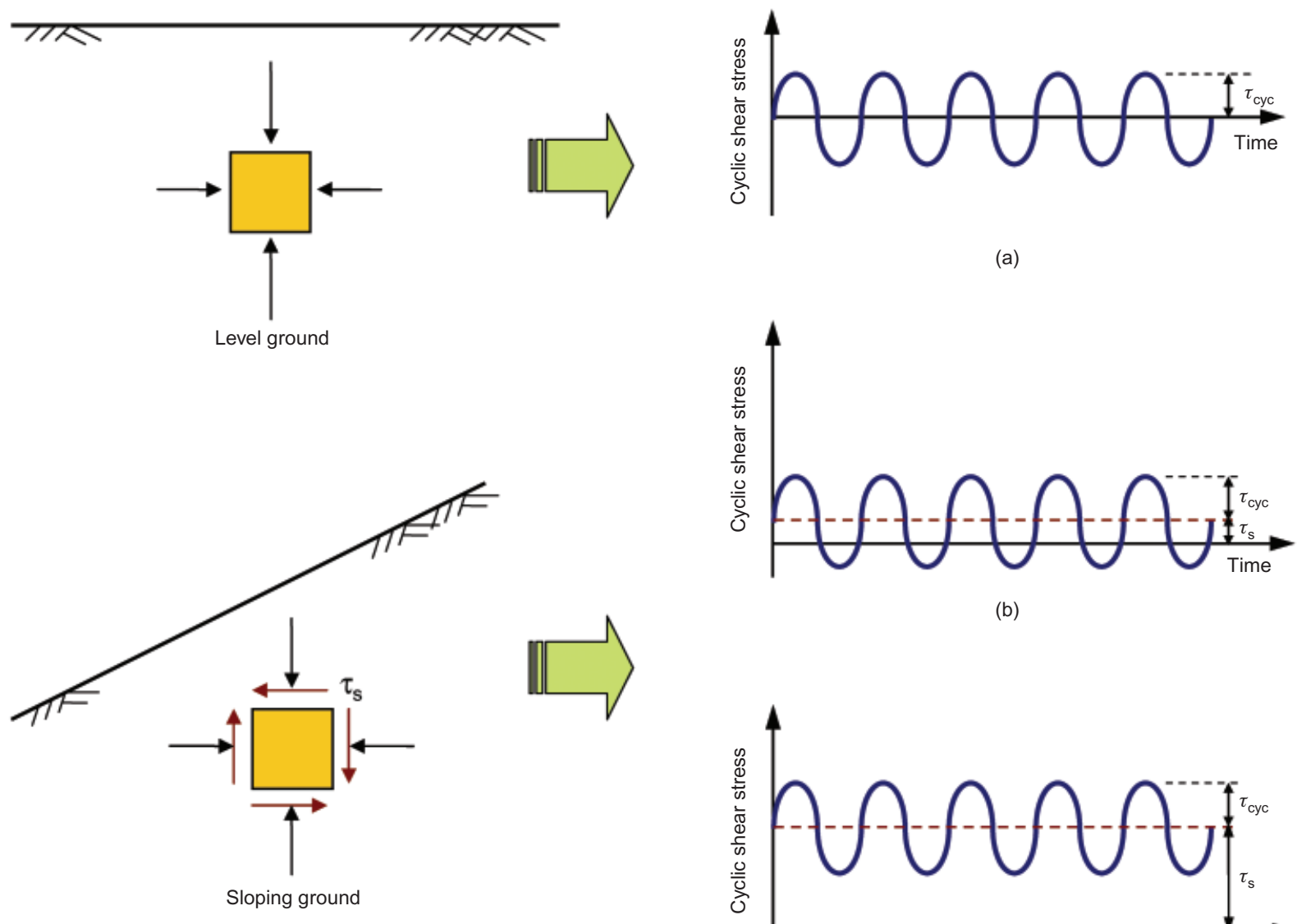

(a)

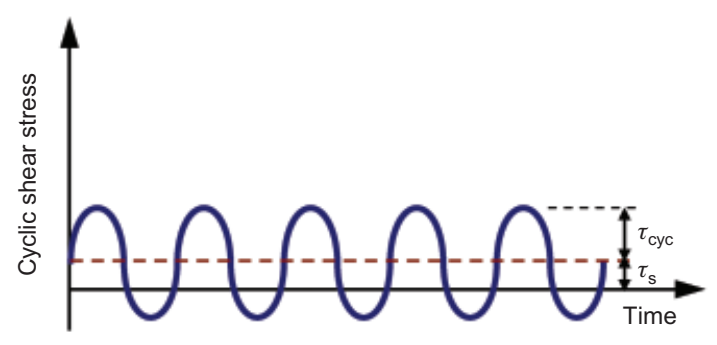

(b)

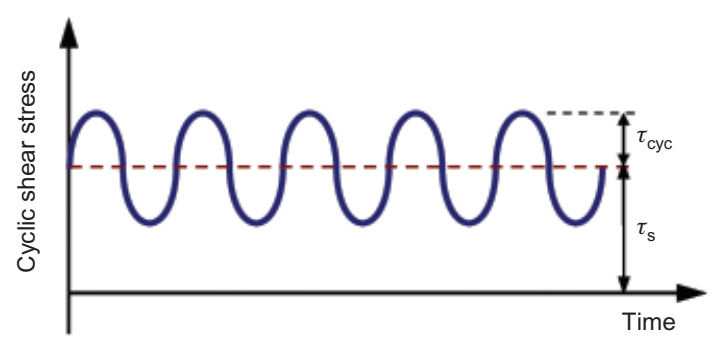

(c)

Fig. 1. Cyclic loading conditions in the laboratory to simulate level ground and sloping ground conditions: (a) symmetrical loading; (b) non-symmetrical loading with stress reversal; (c) non-symmetrical loading without stress reversal

on the plane of interest in the soil sample (i.e. the $45^{\circ}$ plane); superposition of a cyclic shear stress $\tau_{\text {cyc }}$ then produces a cyclic loading that is non-symmetrical about the hydrostatic stress state (Figs 1(b) and 1(c)). It is evident that shear stress reversals will occur if the magnitude of the cyclic shear stress is greater than the initial static shear stress (Fig. 1(b)); otherwise, no stress reversals will appear (Fig. 1(c)).

In such triaxial tests the level of the initial shear stresses can be measured by a parameter $\alpha$, defined as the ratio between the static shear stress $\tau_{\mathrm{s}}$ and the effective normal stress on the $45^{\circ}$ plane, $\sigma_{\mathrm{nc}}^{\prime}$

$$
\alpha=\frac{\tau_{\mathrm{s}}}{\sigma_{\mathrm{nc}}^{\prime}}=\frac{q_{\mathrm{s}}}{2 \sigma_{\mathrm{nc}}^{\prime}}=\frac{\sigma_{1 \mathrm{c}}^{\prime}-\sigma_{3 \mathrm{c}}^{\prime}}{\sigma_{1 \mathrm{c}}^{\prime}+\sigma_{3 \mathrm{c}}^{\prime}}
$$

where $q_{\mathrm{s}}$ is the initial static deviatoric stress. Equation (1) indicates that the level of initial shear stress increases with increasing $\alpha$ values, and the limiting case of $\alpha=0$ represents level ground conditions without initial static shear stresses.

Compared with the extensive work on level ground conditions, there have been only a few experimental studies of the cyclic behaviour of sands in sloping ground conditions. These studies, mainly on triaxial tests and occasionally on simple shear tests, have produced valuable data showing that the presence of initial static shear stresses can have a major effect on the cyclic response of sands. Nevertheless, contradictory opinions appear to exist with respect to this effect. One is that the presence of static shear stresses tends to reduce the rate of pore water pressure generation, and thus increases the resistance to liquefaction (e.g. Seed, 1983); another is, however, that the effect of initial static shear is to reduce the liquefaction resistance (e.g. Yoshimi \& Oh-oka, 1975).

A recent proposal by Harder \& Boulanger (1997), derived from published data and the earlier work of Seed \& Harder (1990), appears to be more reasonable. It states that the effect is positive for dense sand at a relative density of 55$70 \%$, and negative for loose sand at a density of about $35 \%$, with medium dense sand $(45-50 \%)$ being in between. Note that this proposal is limited to the conditions that the effective confining pressure is less than about $300 \mathrm{kPa}$ and the static shear stress ratio $\alpha$ is below $0 \cdot 3$. At the National Center for Earthquake Engineering Research workshop, however, it was recommended that the current proposals 'should not be used by non-specialists' or 'in routine engineering practice' (Youd et al., 2001). This may be due to the scarcity of experimental data and the lack of convergence and consistency in the existing data. Clearly, there is a need for continued research to improve understanding of the complicated static shear stress effects.

Before conducting new research, several observations on the existing data are worth noting. First, most of the experimental work was focused on medium dense to dense sands with a relative density above $35 \%$, and typically $40-60 \%$. Since sands in a looser state are more vulnerable to liquefaction, it is necessary and important to investigate how loose sands behave under different initial stress conditions. Second, the cyclic behaviour of sands has been studied at a relatively low level of initial static shear, with $\alpha$ values up to 0.2 in most cases, and occasionally to $0 \cdot 33$. It is necessary to have $\alpha$ values increased to a higher level, since sands may behave 
differently when the initial shear stress becomes comparable to the cyclic stress applied. Third, it is believed that the behaviour of sands is collectively affected by such major factors as relative density, confining pressure and static shear stress. Much of the early work involved investigation of only one or two of them, varied within a limited range, which may hinder development of an overall understanding. It is important to explore the interdependence of these factors for a wider range of states, so that a comprehensive view can be developed, and the existing inconsistency can be removed.

Keeping the above observations in mind, the goal of the present study is, through a systematic experimental programme, to contribute to the fundamental understanding of sand behaviour under non-symmetrical cyclic loading conditions, and of the initial shear effects in liquefaction resistance. The testing programme has several features, as follows.

(a) It includes a series of cyclic tests on sand specimens with post-consolidation relative density ranging from as low as $10 \%$ to as high as $70 \%$. Specimens with a relative density of $10 \%$ are probably the loosest ever tested under non-symmetrical loading conditions. As will be shown later, they exhibit a significantly different response from that of medium dense and dense sand samples.

(b) It also includes a series of tests under a range of effective confining pressures varying from 100 to $500 \mathrm{kPa}$, and a range of initial shear stresses with $\alpha$ values up to $0 \cdot 6$. As the three major factors are varied in a systematic manner, and over a broad range, the data generated are able to contribute to a fuller understanding of their interrelated effects and, importantly, as will be shown later, to the development of a consistent, critical state view.

Additionally, the systematic database resulting from the present study can be a useful reference for the calibration of advanced constitutive models for sands under non-symmetrical cyclic loading conditions.

\section{EXPERIMENTAL PROGRAMME}

\section{Test material and equipment}

The test material in the study was the Japanese standard sand: Toyoura sand. It is uniform fine sand composed mainly of quartz, with angular to sub-angular grains (Fig. 2). The sand has a mean particle size $\left(D_{50}\right)$ of $0.216 \mathrm{~mm}$ and a uniformity coefficient of 1.392 , with $0 \%$ fines content. Table 1 summarises the physical properties of the sand, and Fig. 2 shows its particle size distribution.

All cyclic tests reported here were performed using an automated triaxial testing system. The system is capable of performing a variety of functions, including isotropic and anisotropic consolidation, and various modes of shear loading under either stress- or strain-controlled conditions, in which all pressures and deformations are regulated and monitored by computer under a closed-loop feedback scheme.

With respect to reproducing field conditions with labora-

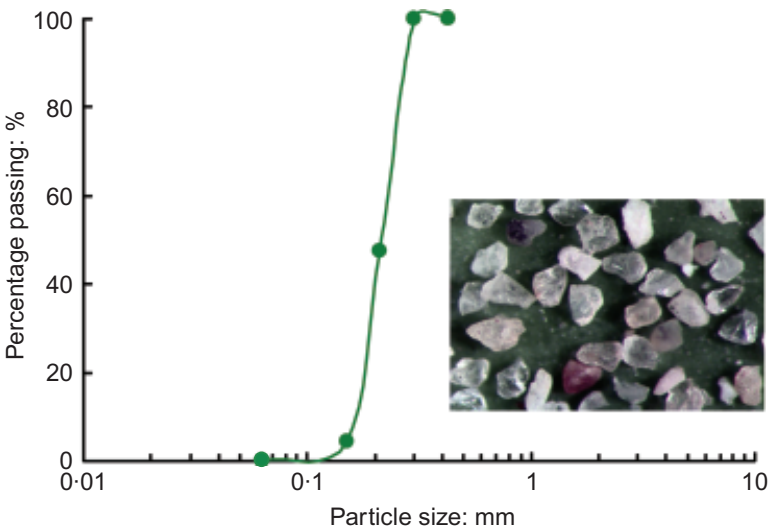

Fig. 2. Test material: microscopic view and particle size distribution

tory element tests, one may consider that the cyclic triaxial test is not as good as the cyclic simple shear test, because of its instantaneous $90^{\circ}$ rotations of the principal stress directions in the loading process. Nevertheless, the triaxial test does have advantages that make it more widely used, including its convenience and reliability in laboratory set-up, and the high repeatability of the test data it generates (Toki et al., 1986; Ishihara, 1993). In particular, triaxial tests have played an important role in the development of the concepts of critical state soil mechanics (Schofield \& Wroth, 1968; Wood, 1990), because the principal stresses in a triaxial setting are clearly defined and measured. In simple shear tests, by contrast, there are problems with the complementary shear stresses on the vertical boundaries of the sample and the unclear lateral stress state, which can cause considerable difficulty in model development.

\section{Sample preparation and saturation}

All sand specimens were prepared using the moist tamping method, since this method is able to produce the widest range in void ratio with satisfactory uniformity (Ishihara, 1996). An oven-dried batch of sand was thoroughly mixed with water and left to stand for about $1 \mathrm{~h}$ for the mixture to reach equilibrium. For each required relative density the predetermined quantity of sand was placed in a mould and then gently compacted into five layers using a flat-bottomed tamper. The concept of undercompaction (Ladd, 1974) was employed to ensure uniformity of specimens. Each specimen so prepared was $71.1 \mathrm{~mm}$ in diameter and $142.2 \mathrm{~mm}$ high.

Complete saturation of specimens is important in the study of soil liquefaction, because the resistance to liquefaction is sensitive to the degree of saturation (Yang, 2002a; Yang et al., 2004). In this study two stages of initial saturation - carbon dioxide flushing and de-aired water flushing - were carried out for each specimen, followed by back-pressure saturation. In most tests a back-pressure of $190 \mathrm{kPa}$ was sufficient to bring the specimen to saturation, with a $B$ value greater than 0.99. Sample dimensions were carefully re-measured after initial saturation to provide in-

Table 1. Physical properties of the test material

\begin{tabular}{l|c|c|c|c|c|c}
\hline $\begin{array}{l}\text { Mean particle size, } \\
D_{50}: \mathrm{mm}\end{array}$ & $\begin{array}{c}\text { Coefficient of } \\
\text { uniformity }\end{array}$ & $\begin{array}{c}\text { Coefficient of } \\
\text { curvature }\end{array}$ & Specific gravity & $\begin{array}{c}\text { Maximum void } \\
\text { ratio, } e_{\max }\end{array}$ & $\begin{array}{c}\text { Minimum void } \\
\text { ratio, } e_{\min }\end{array}$ & Fines content: \% \\
\hline 0.216 & 1.392 & 0.961 & 2.64 & 0.977 & 0.605 & 0 \\
\hline
\end{tabular}


formation on specimen density prior to consolidation, and this information was used to control the density after consolidation. This re-measurement is necessary, especially for loose samples, as a certain amount of volume change may occur during the process of saturation.

\section{Isotropic and anisotropic consolidation}

The consolidation process is important in controlling the initial confining pressure and initial shear stress level in the sand specimen. It is the normal effective stress $\left(\sigma_{\mathrm{nc}}^{\prime}\right)$ and the static shear stress $\left(\tau_{\mathrm{s}}\right)$ on the $45^{\circ}$ plane in the sample that simulate the field conditions. Therefore, when no static shear stresses are present, $\sigma_{\text {nc }}^{\prime}$ is exactly equal to the minor principal consolidation stress $\sigma_{3 \mathrm{c}}^{\prime}$. In cases where initial static shear stresses are present, $\sigma_{\text {nc }}^{\prime}$ needs to be controlled together with $\tau_{\mathrm{s}}$ according to equation (1). In so doing, the principal consolidation stresses $\sigma_{1 \mathrm{c}}^{\prime}$ and $\sigma_{3 \mathrm{c}}^{\prime}$ were applied in segments so that a constant initial static shear stress ratio was maintained in each segment until the required stress conditions were reached. This method of stress control is rather different from that adopted by Vaid \& Chern (1983) and Hyodo et al. (1994), and is considered to be more rational in simulating the static shear effect in cyclic triaxial tests. Because of space limitations, a detailed discussion of this issue will be presented elsewhere.

\section{Shearing with or without stress reversal}

In each test, cyclic shearing was applied to the specimen in a stress-controlled, sinusoidal waveform under undrained conditions. During the shearing process, real-time data were recorded for the deviatoric stress, effective stress, axial strain and volumetric strain. Depending on the relative magnitudes of the static shear stress $\tau_{\mathrm{s}}$ and the applied cyclic shear stress $\tau_{\text {cyc }}$, cyclic loading can occur with or without stress reversals. Note that the cyclic shear stress is related to the cyclic deviatoric stress by $\tau_{\mathrm{cyc}}=q_{\mathrm{cyc}} / 2$.

\section{Test series}

The programme was designed to investigate the combined effects of initial relative density, confining pressure and static shear stress in a systematic manner. In this context, cyclic tests were performed at four initial relative densities ranging from a very loose to a dense state, that is $D_{\text {rc }}=10 \%, 20 \%, 50 \%$ and $70 \%$. The initial relative density here refers to the density after the consolidation stage. This post-consolidation relative density is a more rational indicator for the initial state than the relative density before consolidation. In terms of the relative density prior to consolidation, the value achieved in this study was as low as $5 \%$. For each initial relative density, the initial confining pressure $\sigma_{\text {nc }}^{\prime}$ was varied at three levels $-100,300$ and $500 \mathrm{kPa}$ - which went well beyond the pressure limit in the current proposal of Harder \& Boulanger (1997). Furthermore, at each level of initial confining pressure, tests were conducted by changing the static shear stress and the cyclic shear stress to yield a variety of values of $\alpha$ and degrees of stress reversal. Table 2 summarises the main test series carried out in the study.

\section{OBSERVED BEHAVIOUR AND FAILURE MECHANISMS}

The large number of tests covering a wide range of initial states provides a good opportunity to examine the typical responses and failure mechanisms of sand under both symmetrical and non-symmetrical loading conditions.
Cyclic behaviour of medium dense to dense sand

Figure 3 shows the response of a medium dense specimen at $D_{\mathrm{rc}}=50 \%$ and $\sigma_{\mathrm{nc}}^{\prime}=300 \mathrm{kPa}$ under symmetrical loading conditions (i.e. without initial static shear). This is a typical response, known as cyclic mobility (Castro, 1975). As loading proceeded, excess pore water pressure was gradually built up, and the effective stress was brought toward zero. When the effective stress reached zero momentarily at zero deviatoric stress, initial liquefaction (Seed \& Lee, 1966; Ishihara, 1996) was said to have occurred. Once the deviatoric stress became non-zero, the sand specimen substantially recovered its effective stress and shear stiffness as a result of dilation. This process was repeated in subsequent loading cycles until unacceptably large deformations were reached.

Of particular interest here is how differently the sand will respond if an initial shear stress is applied under otherwise identical conditions. Fig. 4 shows the results of a cyclic test similar to the above, except that an initial static shear stress of $120 \mathrm{kPa}$ was added. In this test, the cyclic stress applied $(155 \mathrm{kPa})$ was greater in magnitude than the initial static stress, leading to the occurrence of stress reversal. For anisotropically consolidated samples the mean effective stress is determined as

$$
p^{\prime}=\sigma_{\mathrm{nc}}^{\prime}-\frac{q_{\mathrm{s}}}{6}=\frac{3-\alpha}{3-3 \alpha} \sigma_{3 \mathrm{c}}^{\prime}
$$

Evidently, in the absence of initial static shear stress, the mean effective stress is exactly the confining pressure $\sigma_{\text {nc }}^{\prime}$ or $\sigma_{3 \mathrm{c}}^{\prime}$.

By comparing Fig. 4 with Fig. 3 it can be seen that the axial strain development tended to be more dominant on the compression side, and the excess pore water pressure was unable to build up to the level of effective confining pressure. A transient strain-softening occurred in the fifth cycle of loading, when the effective stress became close to zero; but once the cyclic stress became non-zero, the sand specimen regained its stiffness substantially (Fig. 4(c)). It is also worth noting that, under non-symmetrical loading conditions, dilation occurred at the very beginning (in the first cycle of compressive loading), and the regain of effective stress due to dilation was much greater in compression than in extension.

To examine further the impact of stress reversal, a sand specimen with the same values of $D_{\mathrm{rc}}$ and $\sigma_{\mathrm{nc}}^{\prime}$ as that given in Fig. 4 was prepared, but it was sheared at a lower cyclic stress level $\left(q_{\mathrm{cyc}}=230 \mathrm{kPa}\right)$, such that no stress reversal occurred. The results are shown in Fig. 5. It is clear that the absence of stress reversal brought about a response significantly different from that with stress reversal. There was not any feature of cyclic mobility in the case of no stress reversal; rather, the axial strain accumulated continuously on the compression side (Fig. 5(b)). It is also of interest to note that, apart from a pronounced strain in the first loading cycle, the strain accumulation in the subsequent loading cycles was at a more or less constant rate (Fig. 5(c)). The test was terminated after about 120 loading cycles, with about $8 \%$ axial strain recorded. The excess pore water pressure at the end of the test was less than $30 \%$ of the effective confining pressure.

As far as denser specimens at $D_{\mathrm{rc}}=70 \%$ are concerned, the tests have indicated that they generally share similar response characteristics with the medium dense specimens at $D_{\mathrm{rc}}=50 \%$, given otherwise identical testing conditions. For the sake of conciseness, the test results are not presented here.

\section{Cyclic behaviour of very loose to loose sand}

There is a lack of experimental data on the cyclic loading behaviour of loose sands with relative density below 30\%. It 
Table 2. Undrained cyclic triaxial test series in this study

\begin{tabular}{|c|c|c|c|c|c|c|}
\hline Series & $\begin{array}{c}\text { Initial density, } \\
D_{\mathrm{rc}}: \%\end{array}$ & $\begin{array}{l}\text { Initial confining } \\
\text { pressure, } \sigma_{\mathrm{nc}}^{\prime}: \mathrm{kPa}\end{array}$ & $\begin{array}{l}\text { Minor principal } \\
\text { stress, } \sigma_{3}^{\prime}: \mathrm{kPa}\end{array}$ & $\begin{array}{c}\text { Initial static } \\
\text { deviatoric stress, } \\
q_{\mathrm{s}}: \mathrm{kPa}\end{array}$ & $\begin{array}{l}\text { Initial static shear } \\
\text { stress ratio, } \alpha\end{array}$ & Loading type \\
\hline \multirow[t]{4}{*}{$\mathrm{I}-\mathrm{a}$} & 10 & 100 & 100 & 0 & 0 & Symmetrical \\
\hline & 10 & 100 & 90 & 20 & $0 \cdot 1$ & Non-symmetrical \\
\hline & 10 & 100 & 75 & 50 & $0 \cdot 25$ & Non-symmetrical \\
\hline & 10 & 100 & 60 & 80 & $0 \cdot 4$ & Non-symmetrical \\
\hline \multirow[t]{4}{*}{$\mathrm{I}-\mathrm{b}$} & 10 & 300 & 300 & 0 & 0 & Symmetrical \\
\hline & 10 & 300 & 270 & 60 & $0 \cdot 1$ & Non-symmetrical \\
\hline & 10 & 300 & 225 & 150 & $0 \cdot 25$ & Non-symmetrical \\
\hline & 10 & 300 & 180 & 240 & $0 \cdot 4$ & Non-symmetrical \\
\hline \multirow[t]{3}{*}{$\mathrm{I}-\mathrm{c}$} & 10 & 500 & 500 & 0 & 0 & Symmetrical \\
\hline & 10 & 500 & 450 & 100 & $0 \cdot 1$ & Non-symmetrical \\
\hline & 10 & 500 & 375 & 250 & $0 \cdot 25$ & Non-symmetrical \\
\hline \multirow[t]{6}{*}{ II-a } & 20 & 100 & 100 & 0 & 0 & Symmetrical \\
\hline & 20 & 100 & 95 & 10 & 0.05 & Non-symmetrical \\
\hline & 20 & 100 & 90 & 20 & $0 \cdot 1$ & Non-symmetrical \\
\hline & 20 & 100 & 75 & 50 & $0 \cdot 25$ & Non-symmetrical \\
\hline & 20 & 100 & 60 & 80 & $0 \cdot 4$ & Non-symmetrical \\
\hline & 20 & 100 & 40 & 120 & $0 \cdot 6$ & Non-symmetrical \\
\hline \multirow[t]{5}{*}{ II-b } & 20 & 300 & 300 & 0 & 0 & Symmetrical \\
\hline & 20 & 300 & 285 & 30 & 0.05 & Non-symmetrical \\
\hline & 20 & 300 & 270 & 60 & $0 \cdot 1$ & Non-symmetrical \\
\hline & 20 & 300 & 225 & 150 & $0 \cdot 25$ & Non-symmetrical \\
\hline & 20 & 300 & 180 & 240 & $0 \cdot 4$ & Non-symmetrical \\
\hline \multirow[t]{5}{*}{ II-c } & 20 & 500 & 500 & 0 & 0 & Symmetrical \\
\hline & 20 & 500 & 475 & 50 & 0.05 & Non-symmetrical \\
\hline & 20 & 500 & 450 & 100 & $0 \cdot 1$ & Non-symmetrical \\
\hline & 20 & 500 & 375 & 250 & $0 \cdot 25$ & Non-symmetrical \\
\hline & 20 & 500 & 300 & 400 & $0 \cdot 4$ & Non-symmetrical \\
\hline \multirow[t]{3}{*}{ III-a } & 50 & 100 & 100 & 0 & 0 & Symmetrical \\
\hline & 50 & 100 & 90 & 20 & $0 \cdot 1$ & Non-symmetrical \\
\hline & 50 & 100 & 60 & 80 & $0 \cdot 4$ & Non-symmetrical \\
\hline \multirow[t]{3}{*}{ III-b } & 50 & 300 & 300 & 0 & 0 & Symmetrical \\
\hline & 50 & 300 & 270 & 60 & $0 \cdot 1$ & Non-symmetrical \\
\hline & 50 & 300 & 180 & 240 & $0 \cdot 4$ & Non-symmetrical \\
\hline \multirow[t]{3}{*}{ III-c } & 50 & 500 & 500 & 0 & 0 & Symmetrical \\
\hline & 50 & 500 & 450 & 100 & $0 \cdot 1$ & Non-symmetrical \\
\hline & 50 & 500 & 300 & 400 & $0 \cdot 4$ & Non-symmetrical \\
\hline \multirow[t]{3}{*}{ IV-a } & 70 & 100 & 100 & 0 & 0 & Symmetrical \\
\hline & 70 & 100 & 90 & 20 & $0 \cdot 1$ & Non-symmetrical \\
\hline & 70 & 100 & 60 & 80 & $0 \cdot 4$ & Non-symmetrical \\
\hline \multirow[t]{3}{*}{ IV-b } & 70 & 300 & 300 & 0 & 0 & Symmetrical \\
\hline & 70 & 300 & 270 & 60 & $0 \cdot 1$ & Non-symmetrical \\
\hline & 70 & 300 & 180 & 240 & $0 \cdot 4$ & Non-symmetrical \\
\hline \multirow[t]{3}{*}{ IV-c } & 70 & 500 & 500 & 0 & 0 & Symmetrical \\
\hline & 70 & 500 & 450 & 100 & $0 \cdot 1$ & Non-symmetrical \\
\hline & 70 & 500 & 300 & 400 & $0 \cdot 4$ & Non-symmetrical \\
\hline
\end{tabular}

is of great interest to examine how different the behaviour of loose sand is from that of medium dense and dense sand. Fig. 6 shows a typical response of a very loose sand specimen at $D_{\mathrm{rc}}=10 \%$ and $\sigma_{\mathrm{nc}}^{\prime}=300 \mathrm{kPa}$ under symmetrical loading conditions. The cyclic deviatoric stress was applied at $75 \mathrm{kPa}$. It can be seen that, although the excess pore water pressure built up gradually and the effective stress was correspondingly reduced as cyclic loading proceeded, there was no notable axial deformation (Fig. 6(b)). When loading was about to enter the eighth cycle, failure was suddenly triggered in the specimen, manifested by runaway deformations and a complete loss of strength. This type of failure was difficult to predict, since the sand, immediately before failure, appeared to be sufficiently strong and stiff, and the axial deformation was negligibly small (Fig. 6(c)). If the same situation were to occur in the field, the soil deposit would fail suddenly, without any warning, and the consequences could thus be disastrous.
Figure 7 shows the results of a test similar to the one above but with an added initial static shear stress of $30 \mathrm{kPa}$. The cyclic deviatoric stress was applied at $95 \mathrm{kPa}$, so that stress reversals occurred during the entire loading process. A similar response, characterised by abrupt runaway deformations, was observed. However, the specimen failed on the compression side (Figs 7(b) and 7(c)) rather than on the extension side as observed in the case of symmetrical loading (Figs 6(b) and (c)). This difference is considered reasonable, because in this test cyclic deviatoric stress was dominated by compressive loading.

Shown in Fig. 8 is the response of a loose specimen at the same initial state $\left(D_{\mathrm{rc}}=10 \%, \sigma_{\mathrm{nc}}^{\prime}=300 \mathrm{kPa}\right)$ as that shown in Fig. 7, but subjected to an initial static shear stress of $75 \mathrm{kPa}$ and a cyclic deviatoric stress of $60 \mathrm{kPa}$, so that no stress reversals occurred. The specimen still showed the abrupt failure pattern as in the symmetrical loading, but the rapid axial strain development was on the 


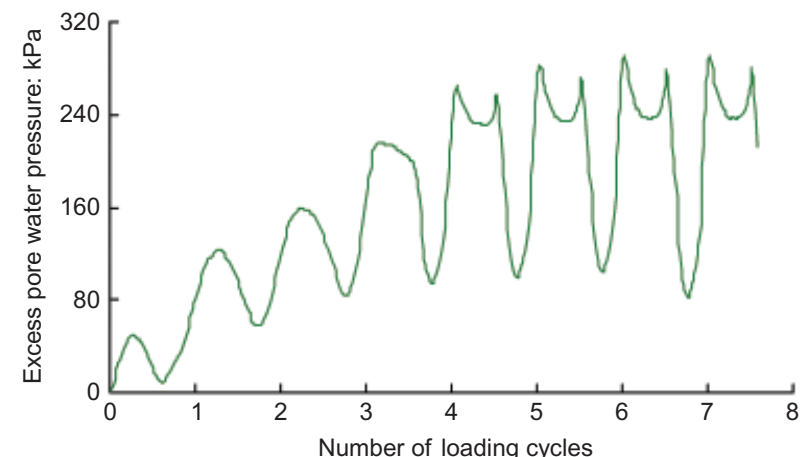

(a)

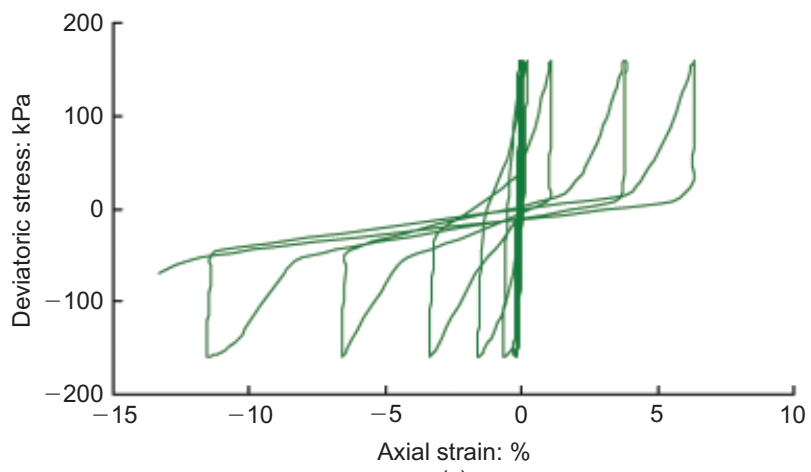

(c)

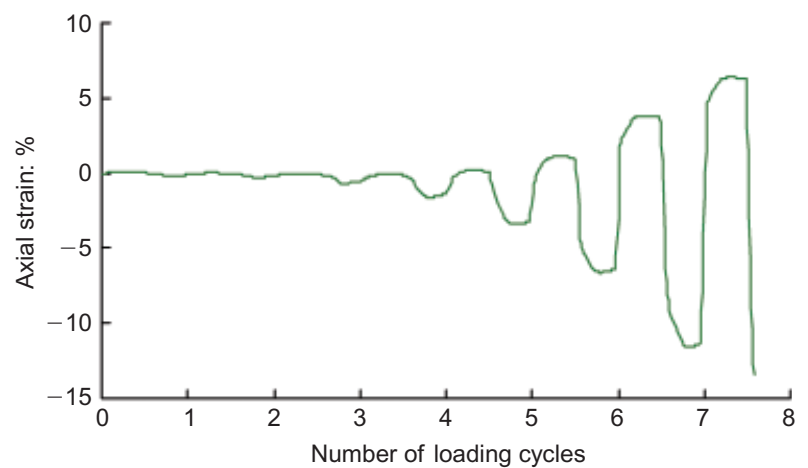

(b)

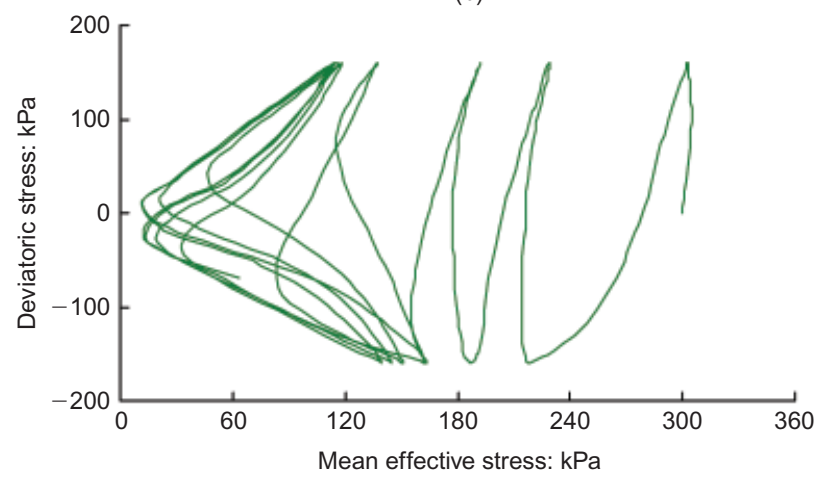

(d)

Fig. 3. Undrained response of medium dense sand under symmetrical cyclic loading $\left(D_{\mathrm{rc}}=50 \%, \sigma_{\mathrm{nc}}^{\prime}=300 \mathrm{kPa}, q_{\mathrm{s}}=0, q_{\mathrm{cyc}}=160 \mathrm{kPa}\right)$

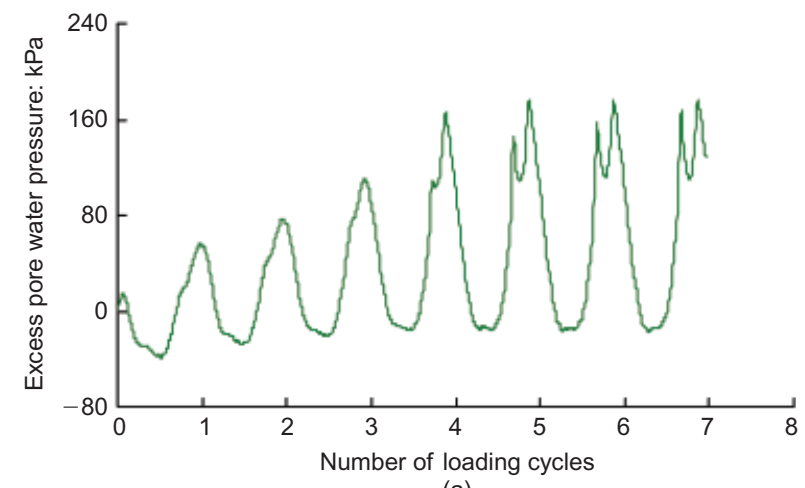

(a)

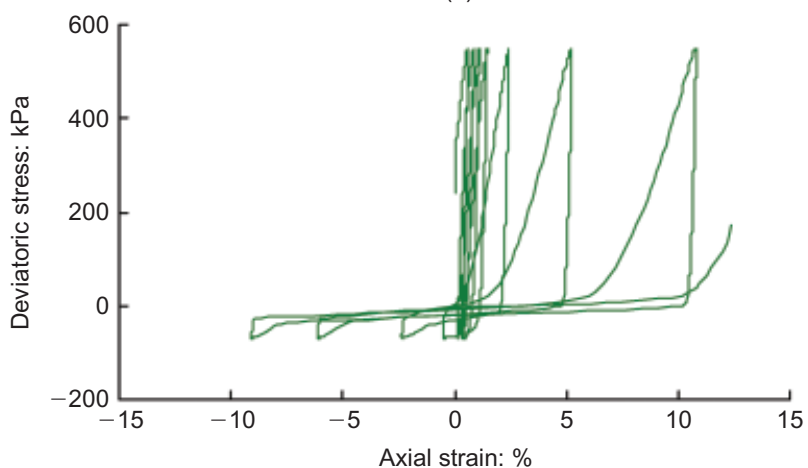

(c)

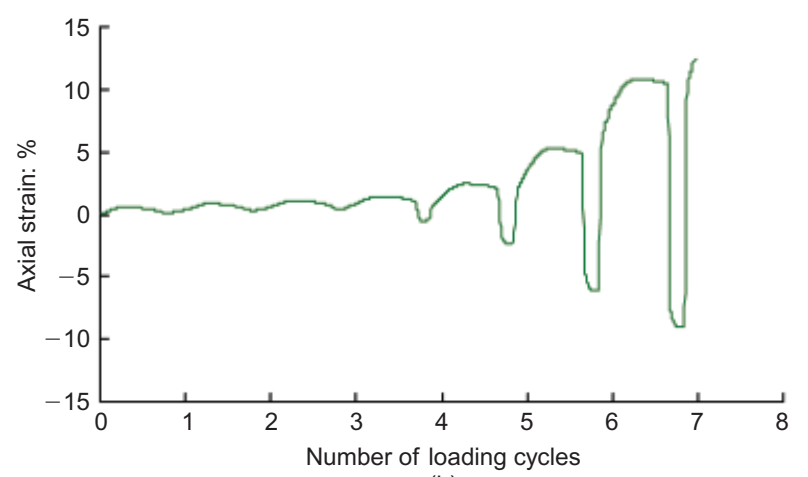

(b)

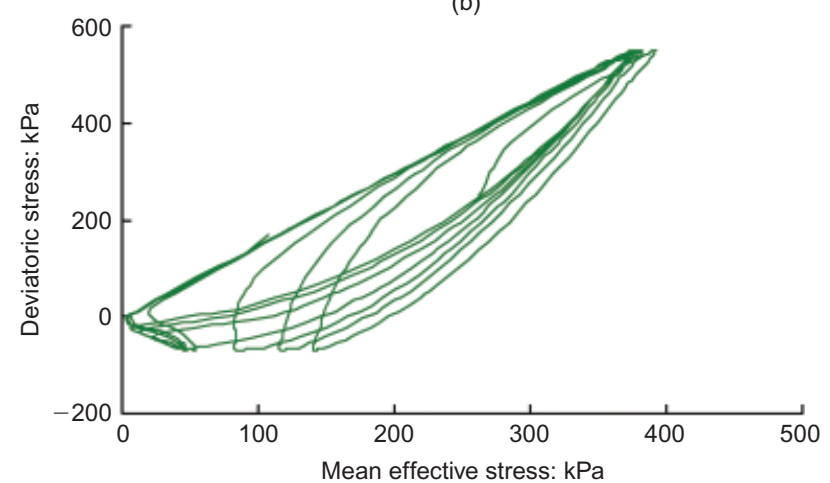

(d)

Fig. 4. Undrained response of medium dense sand under non-symmetrical cyclic loading $\left(D_{\mathrm{rc}}=50 \%, \sigma_{\mathrm{nc}}^{\prime}=300 \mathrm{kPa}, q_{\mathrm{s}}=240 \mathrm{kPa}\right.$, $\left.q_{\mathrm{cyc}}=310 \mathrm{kPa}\right)$

compression side. Recalling the impact of stress reversal on the behaviour of medium dense and dense sand samples, the implication here is that the stress reversal did not impose a marked effect on the response characteristics of loose sand.

\section{Failure modes}

The tests performed in the study have all been thoroughly reviewed, and it has been found that the failure patterns and mechanisms can be categorised into three distinct types, as schematically illustrated in Fig. 9. 


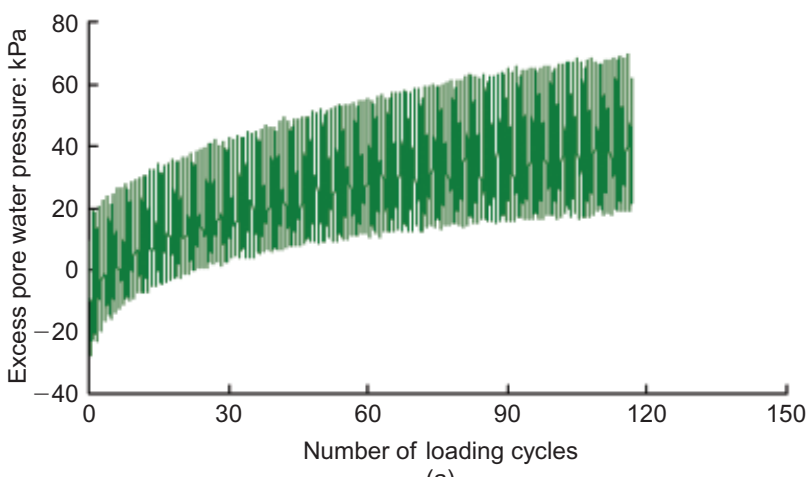

(a)

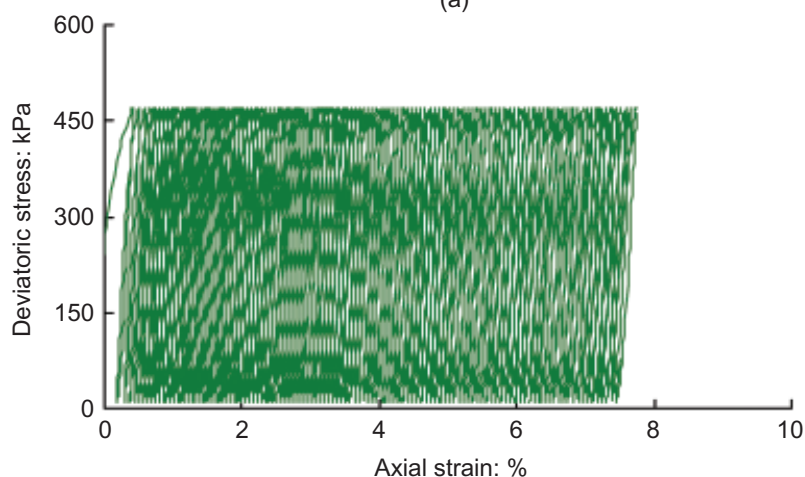

(c)

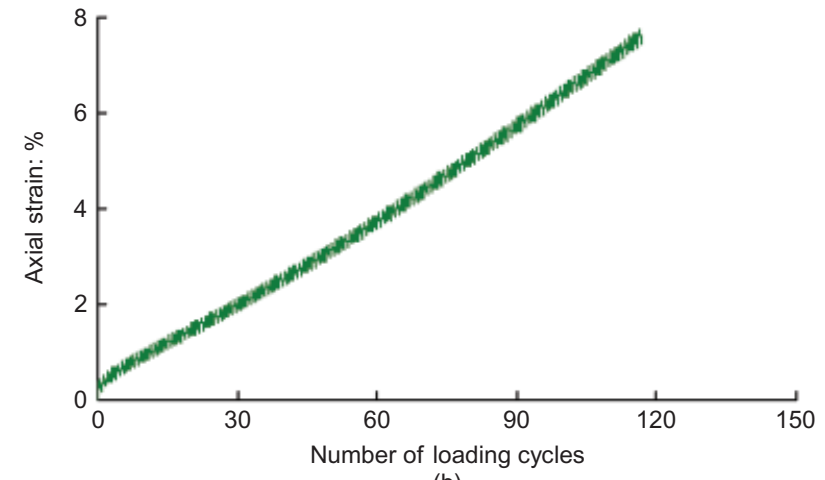

(b)

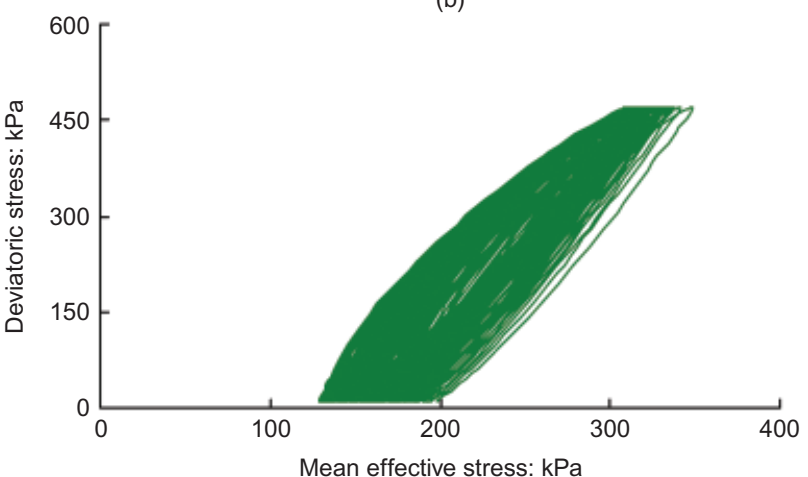

(d)

Fig. 5. Undrained response of medium dense sand under non-symmetrical cyclic loading $\left(D_{\mathrm{rc}}=50 \%, \sigma_{\mathrm{nc}}^{\prime}=300 \mathrm{kPa}, q_{\mathrm{s}}=240 \mathrm{kPa}\right.$, $\left.q_{\mathrm{cyc}}=230 \mathrm{kPa}\right)$

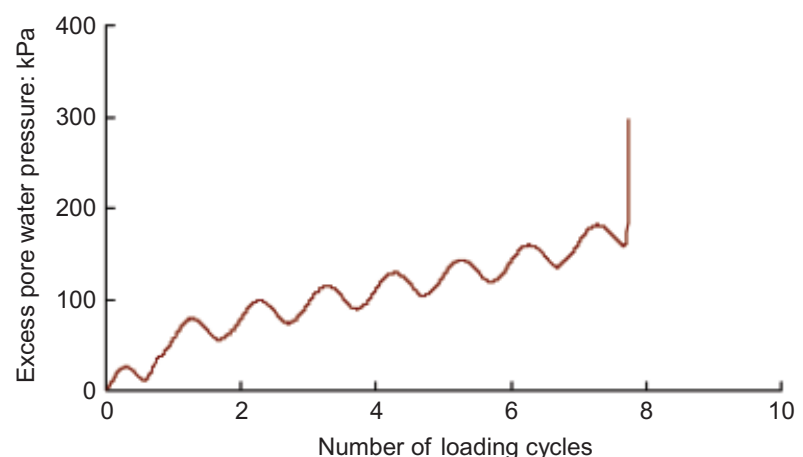

(a)

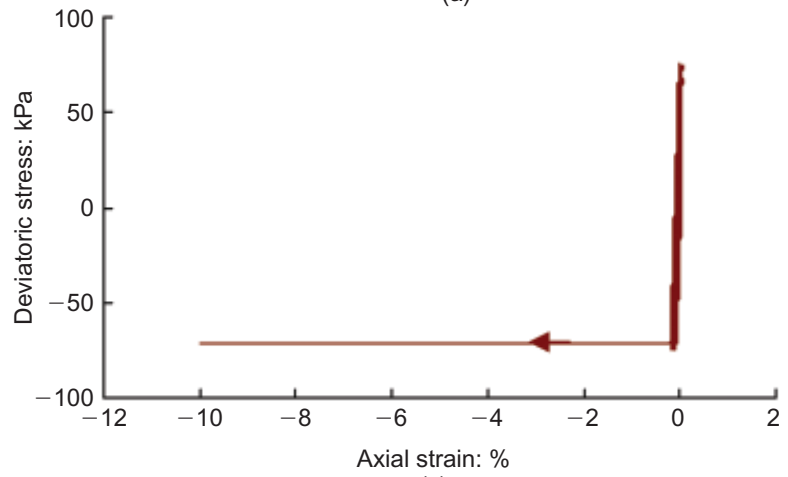

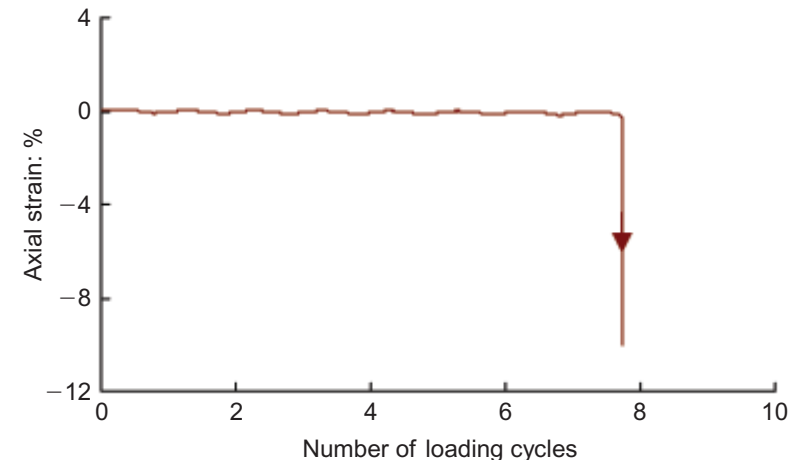

(b)

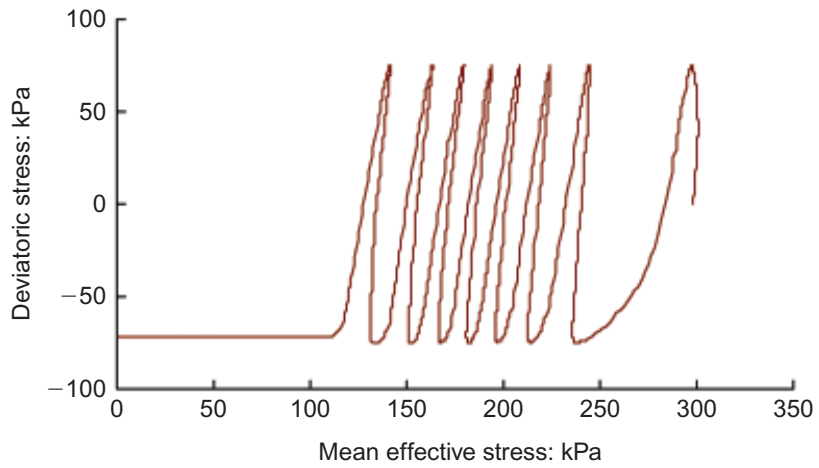

(d)

Fig. 6. Undrained response of loose sand under symmetrical cyclic loading $\left(D_{\mathrm{rc}}=10 \%, \sigma_{\mathrm{nc}}^{\prime}=300 \mathrm{kPa}, q_{\mathrm{s}}=0, q_{\mathrm{cyc}}=75 \mathrm{kPa}\right)$

The first type (Fig. 9(a)) is referred to as flow-type failure or strain-softening, which is pertinent to sand specimens in a very loose to loose state $\left(D_{\mathrm{rc}}=10 \%\right.$ and $\left.20 \%\right)$, and is not affected by stress reversal. This failure mode is featured by a sudden, runaway deformation similar to that observed in loose sand under monotonic loading conditions (e.g. Sladen et al., 1985; Yang, 2002b; Been \& Jefferies, 2004). It is believed that the contractive nature of loose sand facilitates the rapid build-up of pore water pressure and makes it more prone to flow liquefaction.

The second failure mode (Fig. 9(b)), well known as cyclic mobility, has been observed on medium dense and dense 


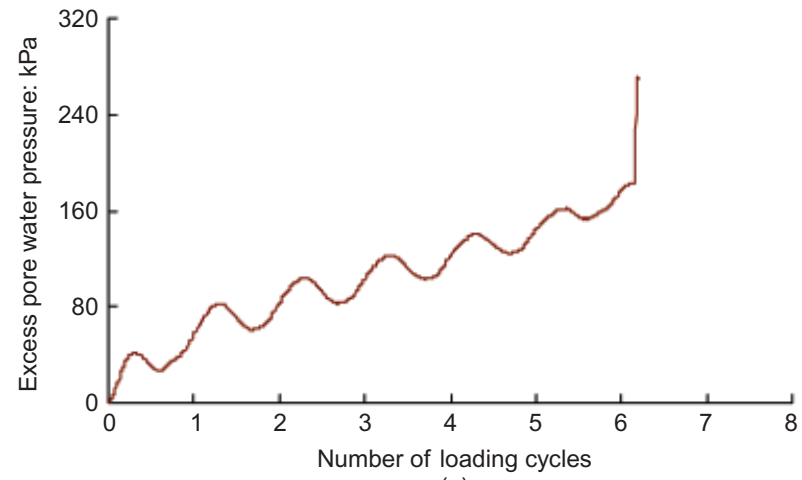

(a)

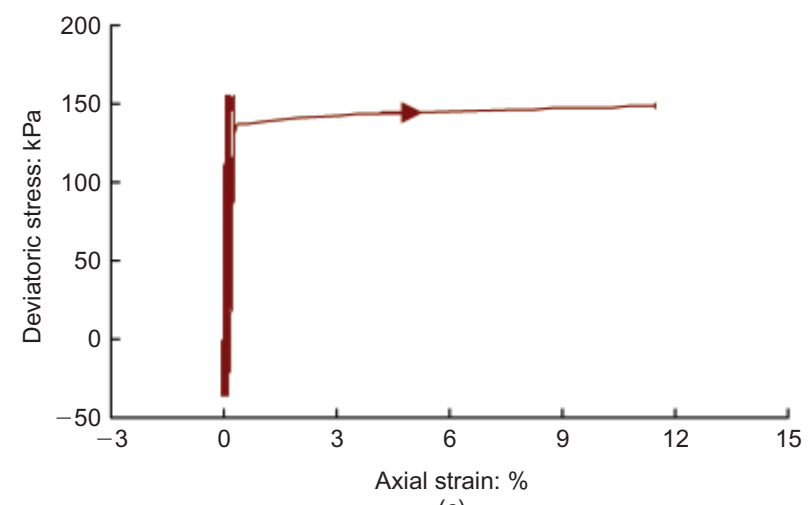

(c)

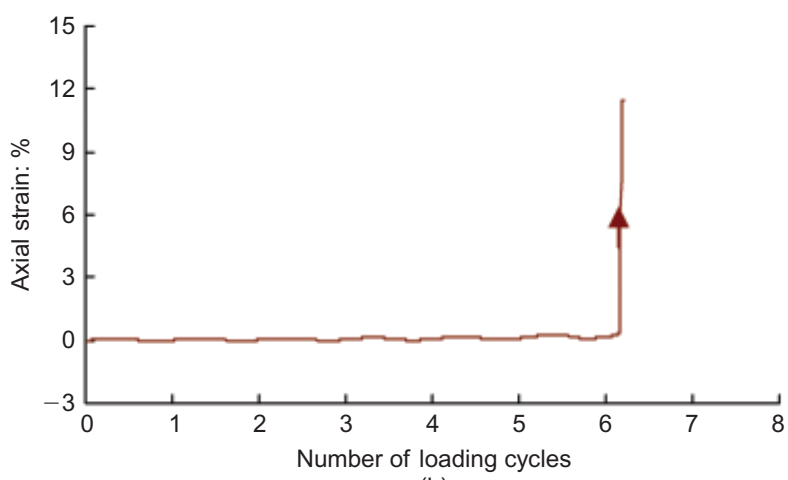

(b)

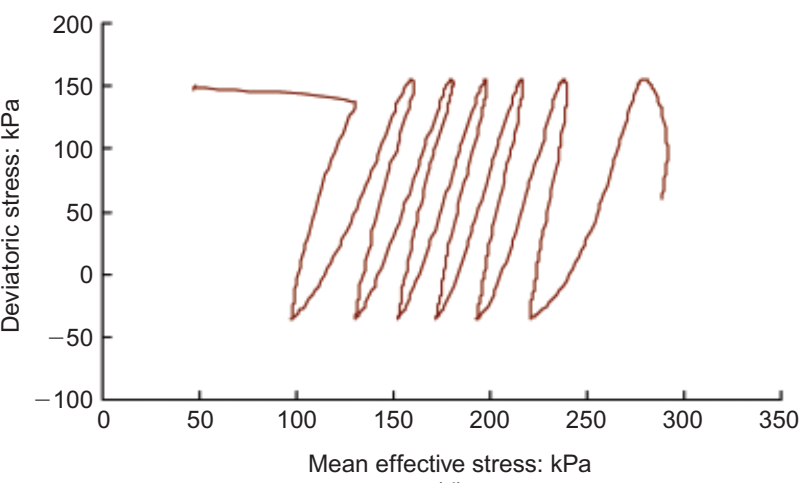

(d)

Fig. 7. Undrained response of loose sand under non-symmetrical cyclic loading $\left(D_{\mathrm{rc}}=10 \%, \sigma_{\mathrm{nc}}^{\prime}=300 \mathrm{kPa}, q_{\mathrm{s}}=60 \mathrm{kPa}\right.$, $\left.q_{\mathrm{cyc}}=95 \mathrm{kPa}\right)$

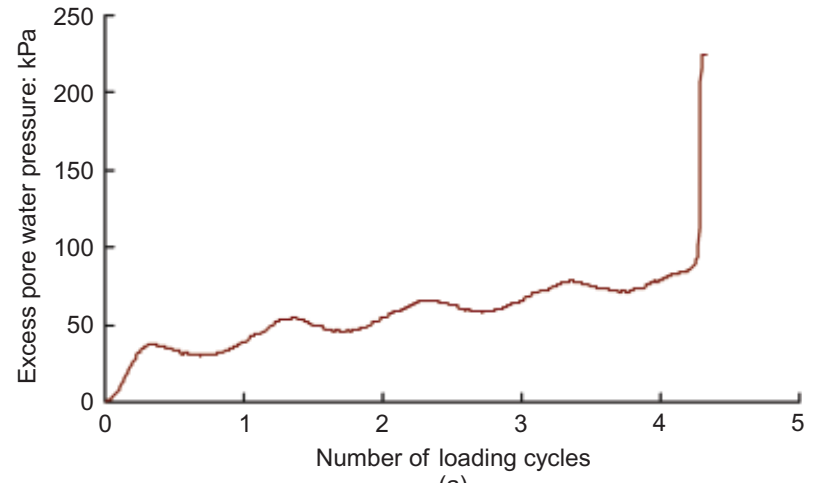

(a)

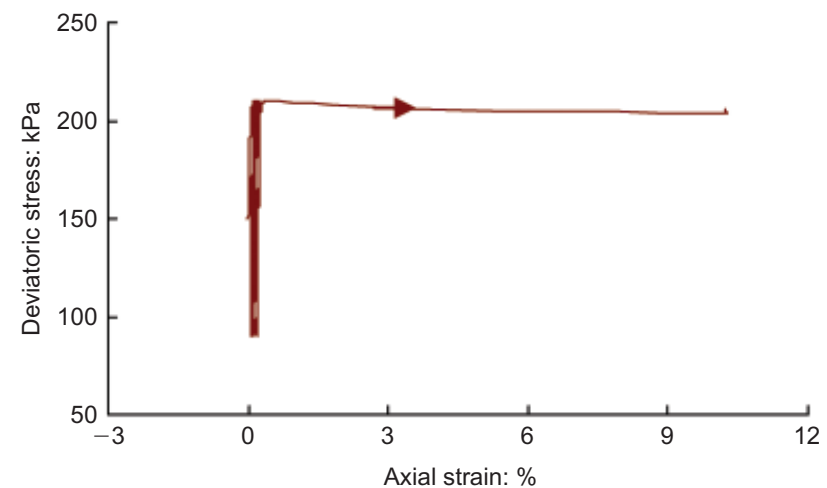

(c)

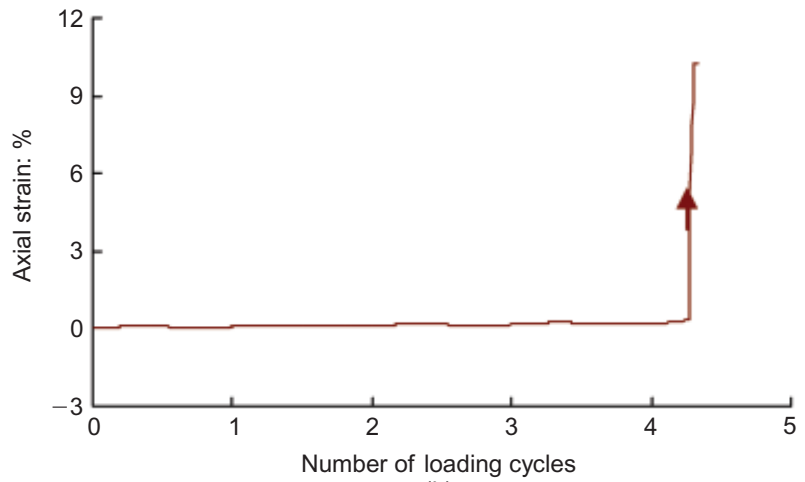

(b)

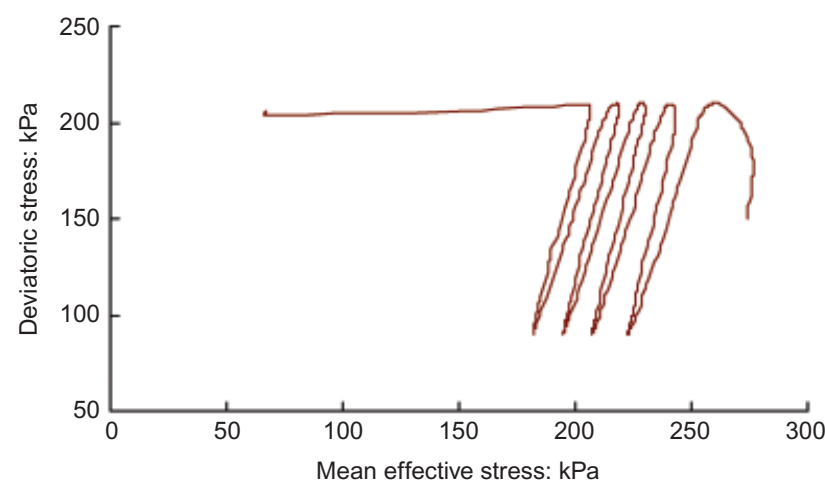

(d)

Fig. 8. Undrained response of loose sand under non-symmetrical cyclic loading $\left(D_{\mathrm{rc}}=10 \%, \sigma_{\mathrm{nc}}^{\prime}=300 \mathrm{kPa}, q_{\mathrm{s}}=150 \mathrm{kPa}, q_{\mathrm{cyc}}=60 \mathrm{kPa}\right)$

samples at $D_{\mathrm{rc}}=50 \%$ and $70 \%$. Apart from the sand being sufficiently dense, another criterion for cyclic mobility to occur is that the loading should be with stress reversal. As discussed before, this condition is dependent on the relative magnitudes of the static shear stress and the applied cyclic shear stress. If the cyclic shear stress is less than the static shear stress, so that no stress reversals occur, the third failure mode, plastic strain accumulation, will become dominant (Fig. 9(c)).

There has been a general belief that stress reversal is a 


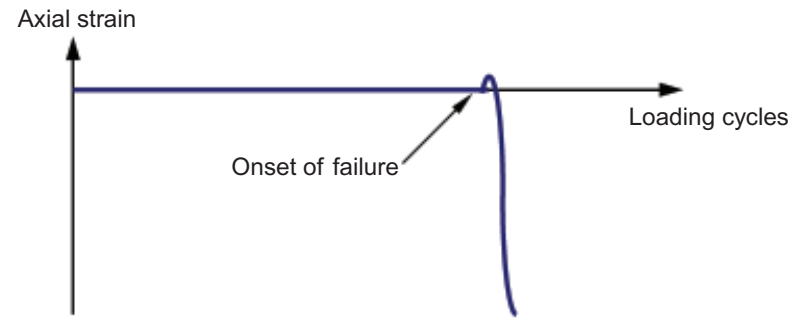

(a)

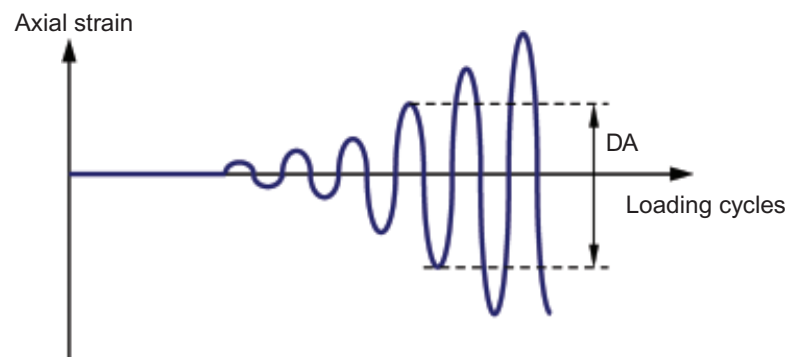

(b)

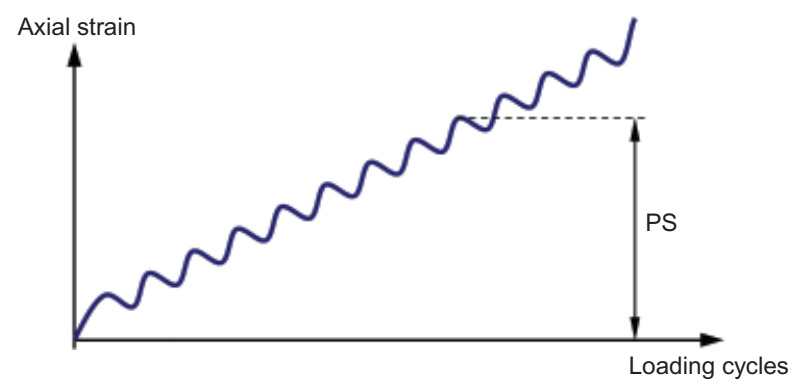

(c)

Fig. 9. Deformation patterns under cyclic loading: (a) flow-type failure; (b) cyclic mobility; (c) accumulated plastic strain

governing factor distinguishing the failure patterns of sand under cyclic loading (Selig \& Chang, 1981). While this belief is confirmed by the tests on medium dense and dense samples in this study, it does not appear to be valid for loose sand specimens at $D_{\mathrm{rc}}=10 \%$ and $20 \%$. The tests have consistently shown that stress reversal was not the key factor governing the failure mechanism of loose sand samples; the failure of these samples was always dominated by the abrupt runaway pattern, regardless of stress reversal. The impact of stress reversal for loose sand appears to lie in whether the excessive axial deformations at failure are in compression or in extension.

To characterise the resistance to cyclic loading, the failure criterion needs to be rationally defined for each type of response. As illustrated in Fig. 9(a), the onset of failure in loose sand can be defined uniquely as the triggering of runaway deformations. However, there is no such unique, definite point indicating the initiation of failure or liquefaction in medium dense and dense sand samples. For the mode of cyclic mobility, the usual approach is to define failure as the point that is accompanied by the occurrence of $5 \%$ double-amplitude (DA) axial strain (Toki et al., 1986; Ishihara, 1996). Although the occurrence of $10 \%$ DA strain is occasionally used as a criterion, 5\% DA is considered to be more reasonable, because it avoids unacceptably large deformations. In the case of plastic strain accumulation response, since the strain development occurs only in one direction, the occurrence of $5 \%$ peak axial strain (PS) in compression becomes as reasonable a criterion as the $5 \%$ DA is for cyclic mobility.

\section{CYCLIC RESISTANCE AND STATE VARIABLES}

The evaluation of cyclic resistance is an important step in the analysis of liquefaction potential in engineering practice. Using the failure criteria defined previously, the cyclic stress ratio $\mathrm{CSR}_{n}$ can be determined for each test as a function of the number of loading cycles to failure

$$
\mathrm{CSR}_{n}=\frac{q_{\mathrm{cyc}}}{2 \sigma_{\mathrm{nc}}^{\prime}}
$$

Figure 10 shows the results for $\mathrm{CSR}_{n}$ for loose samples $\left(D_{\mathrm{rc}}=20 \%\right)$ at various values of $\sigma_{\mathrm{nc}}^{\prime}$ and $\alpha$. For comparison, similar results for medium dense samples $\left(D_{\mathrm{rc}}=50 \%\right)$ are shown in Fig. 11.

Clearly, the initial static shear stress has a significant effect on the position of the $\mathrm{CSR}_{n}$ line, and thus on the liquefaction resistance, and this effect is dependent on the initial relative density $D_{\mathrm{rc}}$ and confining pressure $\sigma_{\mathrm{nc}}^{\prime}$. In what follows, how each of the initial state variables affects the liquefaction resistance, and what their interdependence is, will be investigated in detail. In so doing, the cyclic stress ratio required to cause failure at 10 loading cycles (denoted hereafter as $\mathrm{CRR}_{n}$ ) is introduced to characterise the cyclic resistance of sand.

\section{Effects of relative density and confining pressure}

Figure 12 shows plots of $\mathrm{CRR}_{n}$ against $D_{\mathrm{rc}}$ under a variety of combinations of $\sigma_{\mathrm{nc}}^{\prime}$ and $\alpha$. It can be seen that, no matter how $\sigma_{\text {nc }}^{\prime}$ and $\alpha$ vary, a denser sample always shows a higher value of $\mathrm{CRR}_{n}$ than a looser one under otherwise identical conditions. One of the features of Fig. 12 is that, under a confining pressure of $100 \mathrm{kPa}$ and low static shear stress levels $(\alpha=0$ and $0 \cdot 1)$, the rate of increase in $\mathrm{CRR}_{n}$

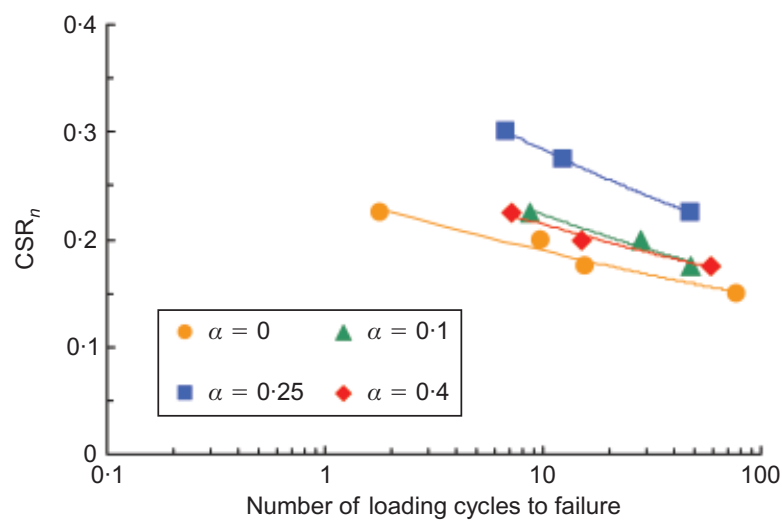

(a)

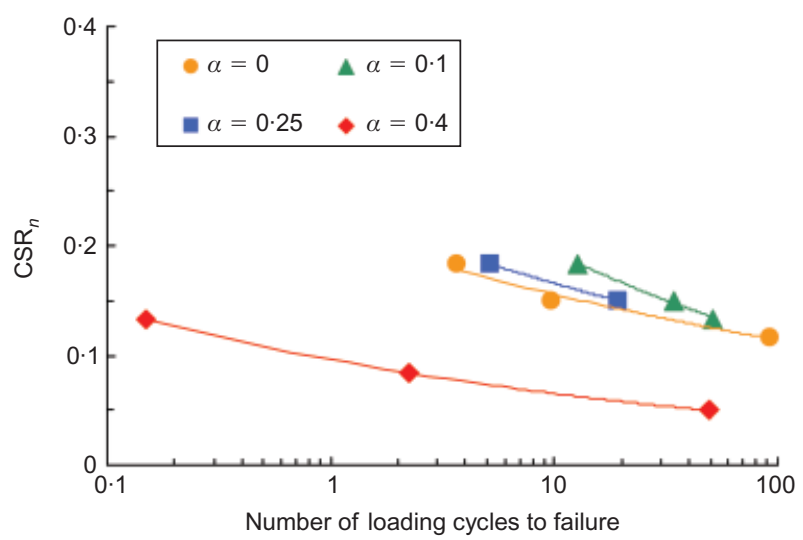

(b)

Fig. 10. Cyclic stress ratio against number of cycles to failure $\left(D_{\mathrm{rc}}=20 \%\right)$ : (a) $\sigma_{\mathrm{nc}}^{\prime}=100 \mathrm{kPa}$; (b) $\sigma_{\mathrm{nc}}^{\prime}=300 \mathrm{kPa}$ 


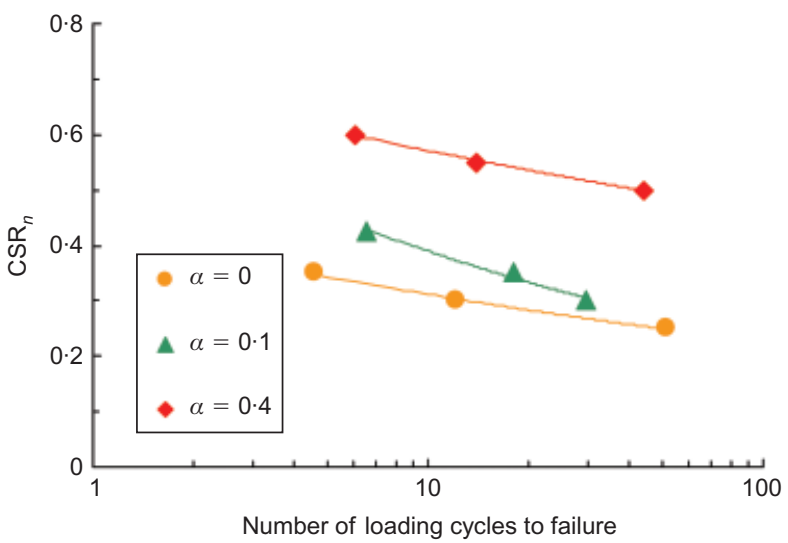

(a)

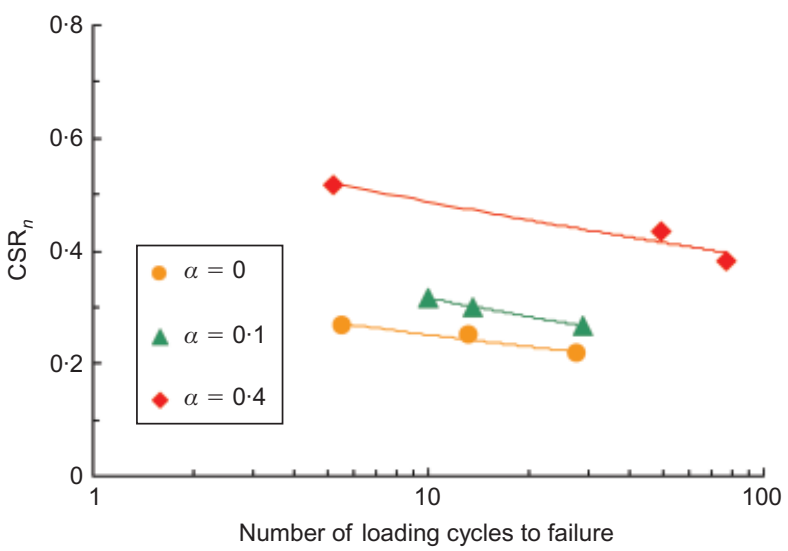

(b)

Fig. 11. Cyclic stress ratio against number of cycles to failure $\left(D_{\mathrm{rc}}=50 \%\right)$ : (a) $\sigma_{\mathrm{nc}}^{\prime}=100 \mathrm{kPa}$; (b) $\sigma_{\mathrm{nc}}^{\prime}=300 \mathrm{kPa}$

resulting from an increase in the relative density from $10 \%$ to $50 \%$ was much smaller than that from $50 \%$ to $70 \%$. This observation implies that, if the sand is originally at a medium dense state, through further densification, its cyclic resistance may be substantially enhanced, but if it is originally in a very loose state, the increase in resistance resulting from densification will be relatively small. In this respect, if in both cases the work done in densification is the same, the effectiveness of densification would be much higher for medium dense sand than for loose sand. However, this effect does not appear to be valid for sand at high static shear stress levels $(\alpha=0 \cdot 4)$ (Fig. 12(c)).

With respect to the effect of confining pressure, Fig. 12 indicates that increasing $\sigma_{\mathrm{nc}}^{\prime}$ always reduces the value of $\mathrm{CRR}_{n}$, as long as $D_{\mathrm{rc}}$ and $\alpha$ remain unchanged. The important implication is that the already low resistance the loose sand bears would be further reduced by increasing the confining stress level. This impact may become crucial for high initial shear stress levels. For example, at $\alpha=0.4$ the sand specimen with $D_{\text {rc }}=20 \%$ had a $\mathrm{CRR}_{n}$ of 0.2 under $\sigma_{\mathrm{nc}}^{\prime}=100 \mathrm{kPa}$, but it tended to lose its resistance completely when $\sigma_{\mathrm{nc}}^{\prime}$ was raised to $500 \mathrm{kPa}$. One more thing worth noting in Fig. 12 is that increasing $\sigma_{\mathrm{nc}}^{\prime}$ from $100 \mathrm{kPa}$ to $300 \mathrm{kPa}$ tends to reduce the beneficial effect of increasing $D_{\text {rc }}$, particularly for medium dense to dense sand, implying the interdependence of the effects of relative density and confining pressure.

The effect of high confining stress may be characterised by introducing a so-called $K_{\sigma}$ factor (Seed, 1983; Youd et al., 2001). Before doing that, the rationale behind the current definition for $K_{\sigma}$ needs to be carefully examined, and some modification is required. Given the space limitations, discussion of this will be presented in a future paper.

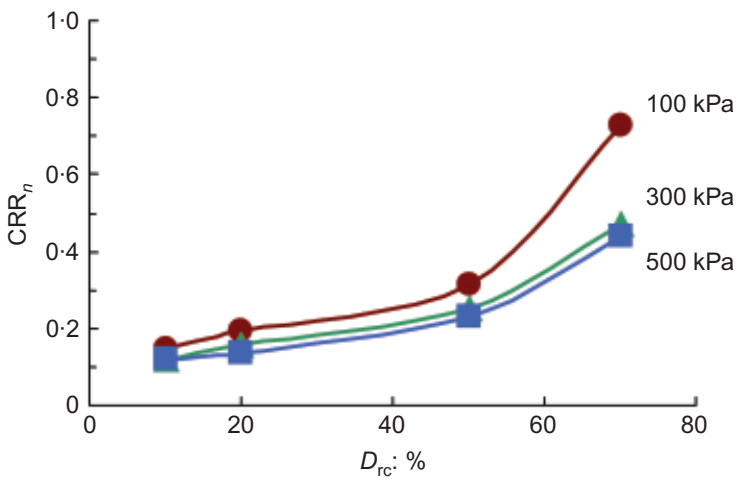

(a)

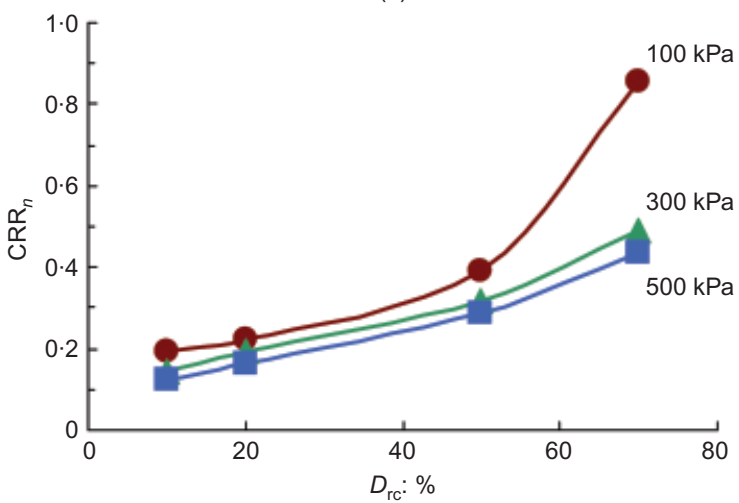

(b)

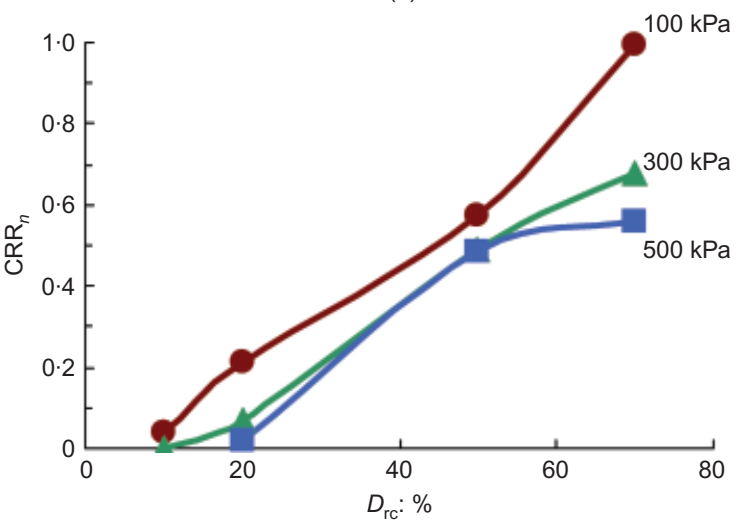

(c)

Fig. 12. Effects of relative density and confining pressure on cyclic resistance: (a) no initial shear $(\alpha=0)$; (b) small initial shear $(\alpha=0 \cdot 1)$; (c) large initial shear $(\alpha=0 \cdot 4)$

\section{Effects of initial static shear}

Figure 13 presents values of $\mathrm{CRR}_{n}$ against $\alpha$ for a variety of initial states in terms of $D_{\mathrm{rc}}$ and $\sigma_{\mathrm{nc}}^{\prime}$. A marked feature is that $\alpha$ affects the cyclic resistance differently in loose sand and dense sand. In general, for the entire range of confining pressures tested, $\mathrm{CRR}_{n}$ tends to increase and then decrease with increasing values of $\alpha$ in loose sand specimens ( $D_{\mathrm{rc}}=10 \%$ and $20 \%$ ), but it continues to increase with $\alpha$ in medium dense and dense sand samples $\left(D_{\mathrm{rc}}=50 \%\right.$ and $70 \%)$.

To characterise this effect, a factor denoted by $K_{\alpha}$ is introduced by following the proposal of Seed (1983). It is defined here as the ratio of $\mathrm{CRR}_{n}$ at any $\alpha$ to that at $\alpha=0$ under a fixed $\sigma_{\mathrm{nc}}^{\prime}$

$$
K_{\alpha}=\frac{\mathrm{CRR}_{\sigma, \alpha}}{\mathrm{CRR}_{\sigma, 0}}
$$

Evidently, the variation of $K_{\alpha}$ is the direct result of varying 


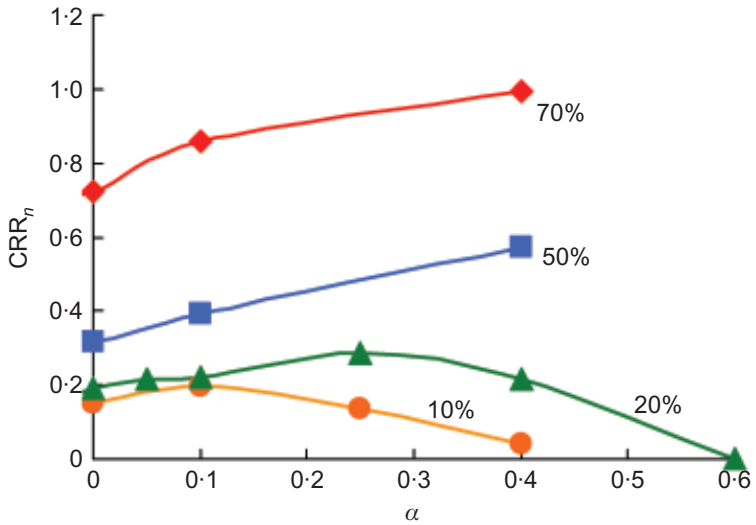

(a)

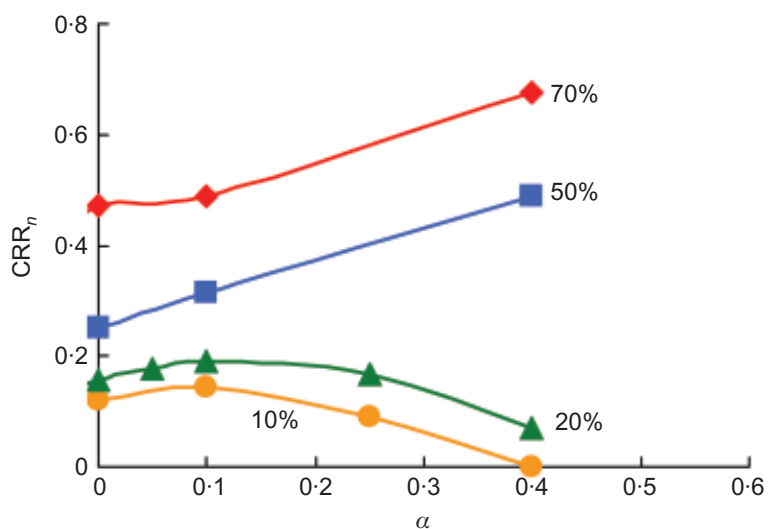

(b)

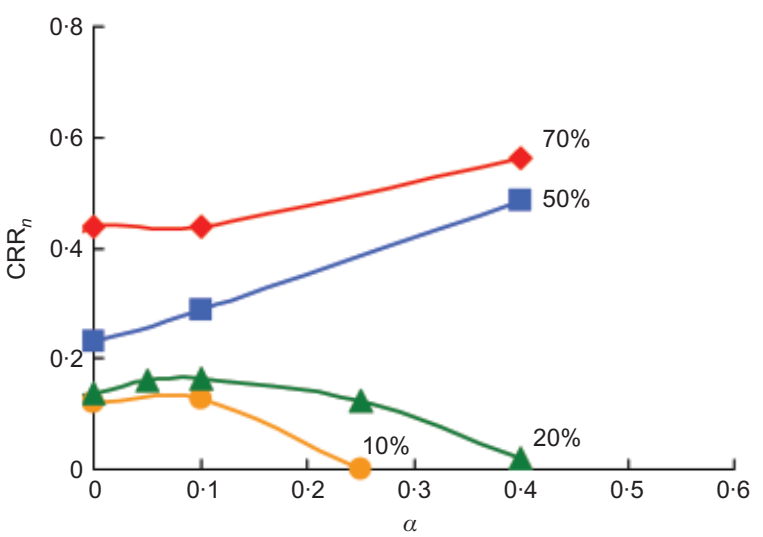

(c)

Fig. 13. Cyclic resistance as a function of the level of initial shear stress: (a) $\sigma_{\mathrm{nc}}^{\prime}=100 \mathrm{kPa}$; (b) $\sigma_{\mathrm{nc}}^{\prime}=300 \mathrm{kPa}$; (c) $\sigma_{\mathrm{nc}}^{\prime}=$ $500 \mathrm{kPa}$

$\mathrm{CRR}_{n}$ with $\alpha$, and when $\alpha=0, K_{\alpha}$ is always equal to $1 . K_{\alpha}$ does not indicate exactly what cyclic resistance the sand bears; rather, it indicates how great the change in cyclic resistance is due to the presence of a certain level of initial shear stress.

Typical plots of $K_{\alpha}$ against $\alpha$ are shown in Fig. 14 for various values of $D_{\mathrm{rc}}$ and $\sigma_{\mathrm{nc}}^{\prime}$. For loose sand specimens the presence of initial static shear stress can be beneficial (i.e. $\left.K_{\alpha}>1\right)$ or detrimental $\left(K_{\alpha}<1\right)$; but for medium dense and dense specimens the effect appears to be always beneficial. However, as will be discussed later in a theoretical framework, the effect of initial static shear stress may also become negative for sand at high relative density under some circumstances, and a unified view can be established for both loose and dense sand samples.

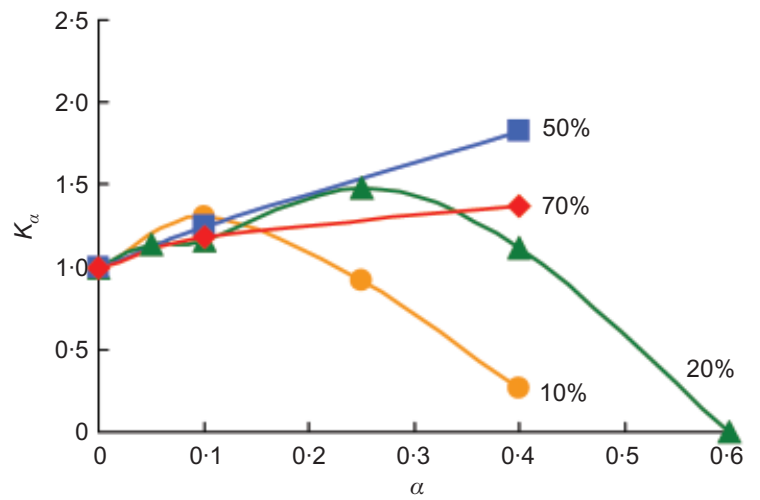

(a)

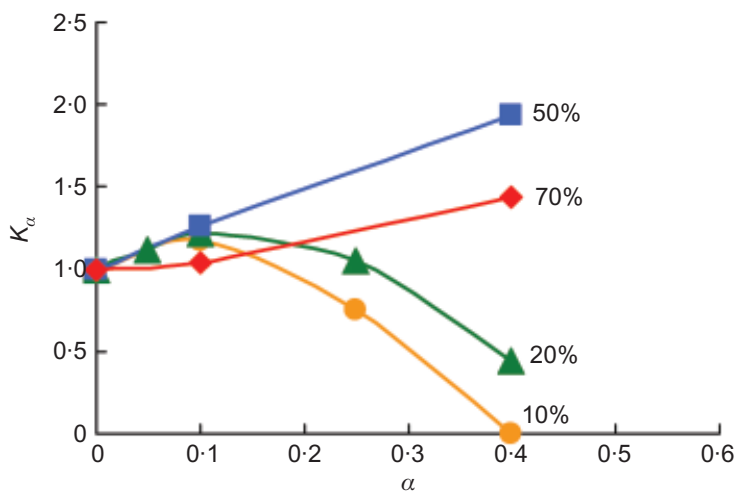

(b)

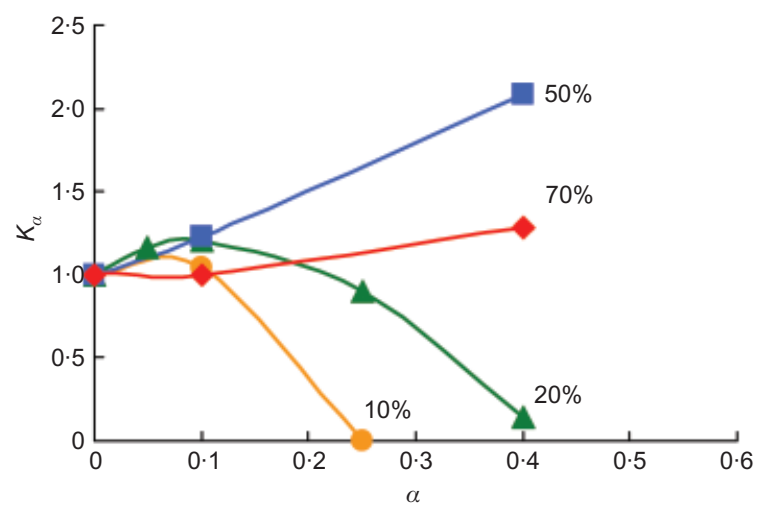

(c)

Fig. 14. Effects of confining pressure and relative density on the relationship between $K_{\alpha}$ and $\alpha$ : (a) $\sigma_{\mathrm{nc}}^{\prime}=100 \mathrm{kPa}$; (b) $\sigma_{\mathrm{nc}}^{\prime}=$ $300 \mathrm{kPa}$; (c) $\sigma_{\mathrm{nc}}^{\prime}=500 \mathrm{kPa}$

\section{CRITICAL-STATE-BASED VIEWS}

Critical state soil mechanics (CSSM) has been shown to be a useful conceptual framework for describing the monotonic behaviour of sand (e.g. Been \& Jefferies, 1985; Wood et al., 1994; Yang \& Li, 2004). The CSSM framework appears to have received less attention for cyclic loading behaviour of sand. Recently, Boulanger (2003) made a good attempt to relate $K_{\alpha}$ values to a relative state parameter index. The focus here is on exploring whether the cyclic resistance and interrelated effects of various state variables could be accounted for in the CSSM framework. A useful concept, termed threshold $\alpha$, will be developed, along with the use of a no-stress-reversal line, and the dependence of the threshold $\alpha$ on a state parameter will be established.

The central point of the CSSM framework is that there is an ultimate state of shear failure at which the sand deforms continuously under constant stress and constant volume. Fig. 15 shows the critical state line (CSL) of Toyoura sand and the initial states of all tests performed in this study in the 


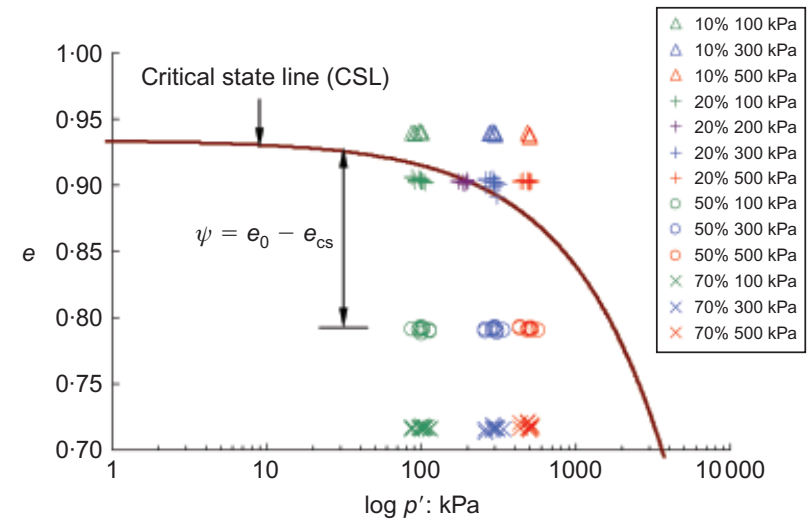

Fig. 15. Initial states of specimens with reference to the critical state line

$e-\log p^{\prime}$ plane, where $e$ is the void ratio and $p^{\prime}$ is the mean effective stress. The CSL of Toyoura sand is reproduced from the undrained monotonic triaxial tests conducted by Verdugo \& Ishihara (1996).

It can be seen from Fig. 15 that the initial states of specimens at $D_{\mathrm{rc}}=50 \%$ and $70 \%$ all lie below the CSL; in the CSSM concept, these samples should behave in a dilative manner. All specimens at a relative density of $10 \%$ and most specimens at $20 \%$ will show contractive behaviour, because their states are above the CSL. The 20\% sand specimens at a mean effective stress of about $200 \mathrm{kPa}$ may tend to exhibit a marginal response.

\section{Cyclic resistance against state parameter}

The state parameter proposed by Been \& Jefferies (1985) is adopted to account for the initial state in terms of relative density and stress level. It is defined as

$$
\psi=e_{0}-e_{\mathrm{cs}}
$$

where $e_{0}$ is the initial void ratio of the sample and $e_{\mathrm{cs}}$ is the void ratio of the critical state at the same mean stress (Fig. 15). Obviously, the parameter is a measure of how far the current state is from the critical state. If $\psi$ is negative, the sand is considered to be in a dense, dilative state, whereas it is in a loose, contractive state if $\psi$ is positive.

Figure 16(a) shows the values of $\mathrm{CRR}_{n}$ against the state parameter $\psi$ for the tests performed. A striking feature of this plot is that there is a fairly good trend between $\mathrm{CRR}_{n}$ and the state parameter, showing that $\mathrm{CRR}_{n}$ tends to decrease as $\psi$ increases: that is, the sand will bear a higher cyclic resistance when it becomes denser and/or when it is subjected to a lower confining pressure.

For the purpose of comparison, the relative state parameter proposed by Konrad (1988) is adopted as well, to describe the initial state. This relative state parameter is defined as

$$
\psi_{1}=\frac{e_{0}-e_{\mathrm{cs}}}{e_{\max }-e_{\min }}
$$

where $e_{\max }$ and $e_{\min }$ are the maximum and minimum void ratios of a sand. Fig. 16(b) shows $\mathrm{CRR}_{n}$ against $\psi_{1}$ for the tests performed. The trend is almost the same as that in Fig. 16(a). In the following discussion, only the state parameter $\psi$ will be the focus.

In Fig. 16(a), at each value of $\psi$ there is a range of data points scattering vertically. This scatter is the result of varying $\alpha$ at each set of initial states in terms of $D_{\mathrm{rc}}$ and $\sigma_{\text {nc }}^{\prime}$. By re-plotting the data in terms of $\alpha$, an improved

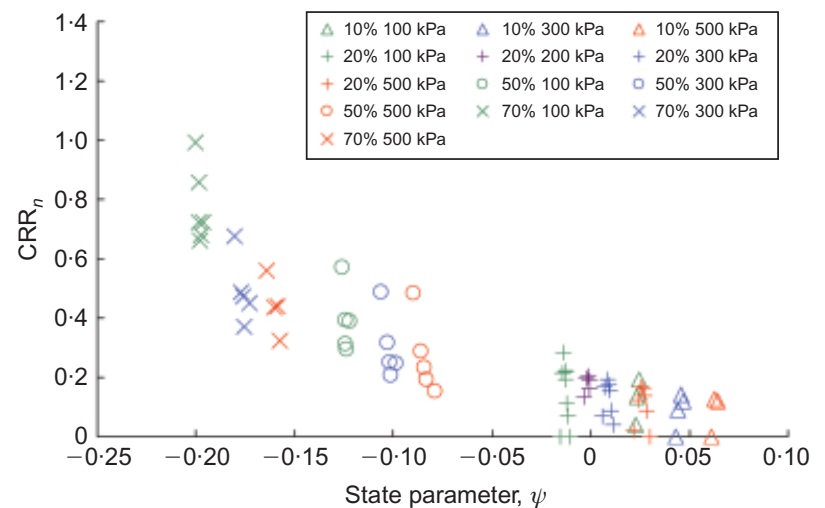

(a)

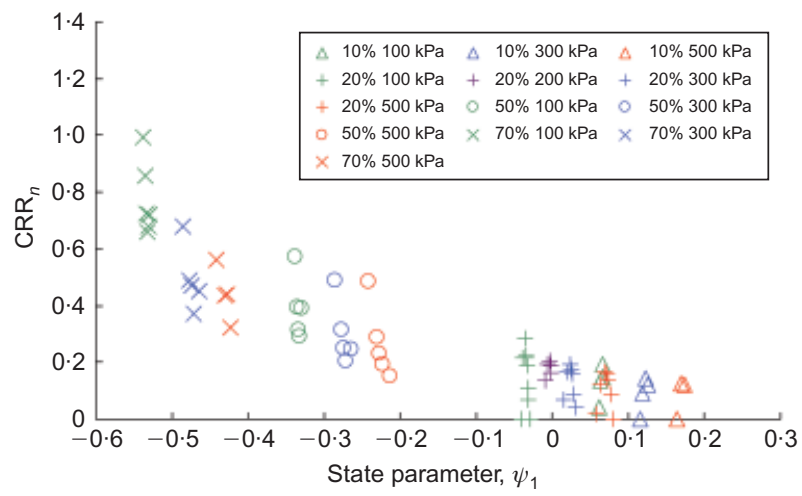

(b)

Fig. 16. Cyclic resistance as a function of: (a) state parameter; (b) relative state parameter

correlation is achieved between $\mathrm{CRR}_{n}$ and $\psi$, as shown in Fig. 17.

Based on the data points in Fig. 17, and for the first instance, a best-fit line can be drawn to represent the variation of $\mathrm{CRR}_{n}$ with $\psi$. If a non-linear relationship is assumed, one may arrive at the form

$$
C R R_{n}=8 \cdot 1508 \psi^{2}-1 \cdot 2895 \psi+0 \cdot 1498
$$

If a linear relationship is assumed, then

$$
C R R_{n}=-2 \cdot 3794 \psi+0 \cdot 1719
$$

\section{Threshold $\alpha$}

Referring back to Fig. 13, where cyclic resistance is plotted as a function of $\alpha$, all loose samples show an increase and then a decrease in $\mathrm{CRR}_{n}$ with increasing values of $\alpha$. This observation suggests that a threshold value of $\alpha$ exists: when $\alpha$ reaches this value, the cyclic resistance tends to reduce with further increase in $\alpha$. Furthermore, by recalling the role of stress reversal, a no-stress-reversal line is introduced herein as the line dividing between loading with stress reversals and loading without stress reversals. By adding this line to the plot of $\mathrm{CRR}_{n}$ against $\alpha$, as shown in Fig. 18 for the case of $D_{\text {rc }}=20 \%$, it is of interest to note that whenever the $\mathrm{CRR}_{n}$ trend line touches the no-reversal line, $\mathrm{CRR}_{n}$ is about to decrease with increase in $\alpha$. In other words, when $\alpha$ is increased to a level such that the loading required to bring the sand to failure is without stress reversals, then the cyclic resistance starts to reduce.

Apparently, the use of the no-stress-reversal line helps in determining the threshold $\alpha$ in a rational way. One key point to note is that this threshold $\alpha$ is a function of the relative density and initial confining pressure. This can be observed in Figs 13 and 18, which indicate that the plot of $\mathrm{CRR}_{n}$ 


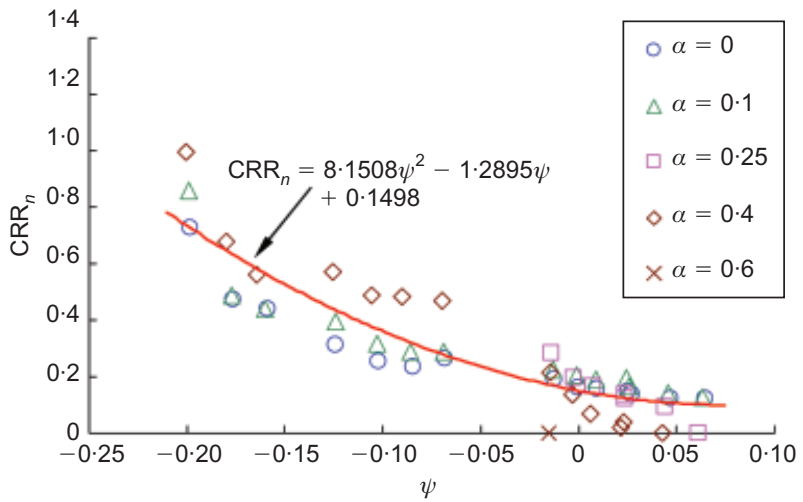

(a)

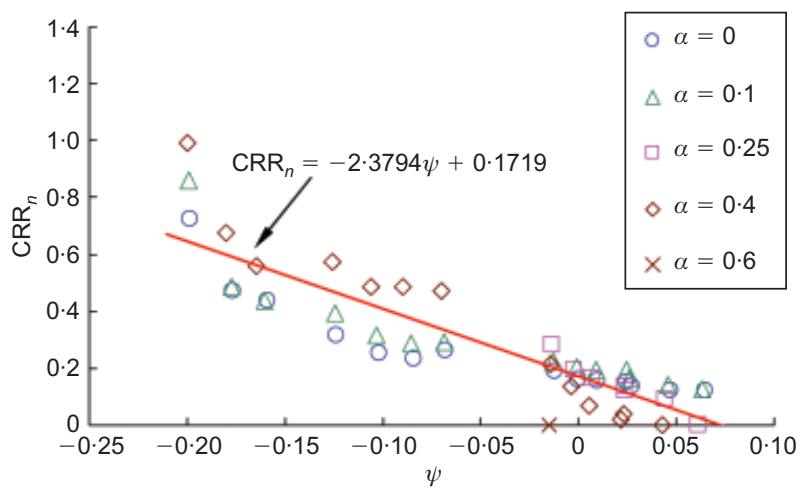

(b)

Fig. 17. Correlation between $\mathrm{CRR}_{n}$ and $\psi$ for various $\alpha$ values: (a) non-linear representation; (b) linear representation

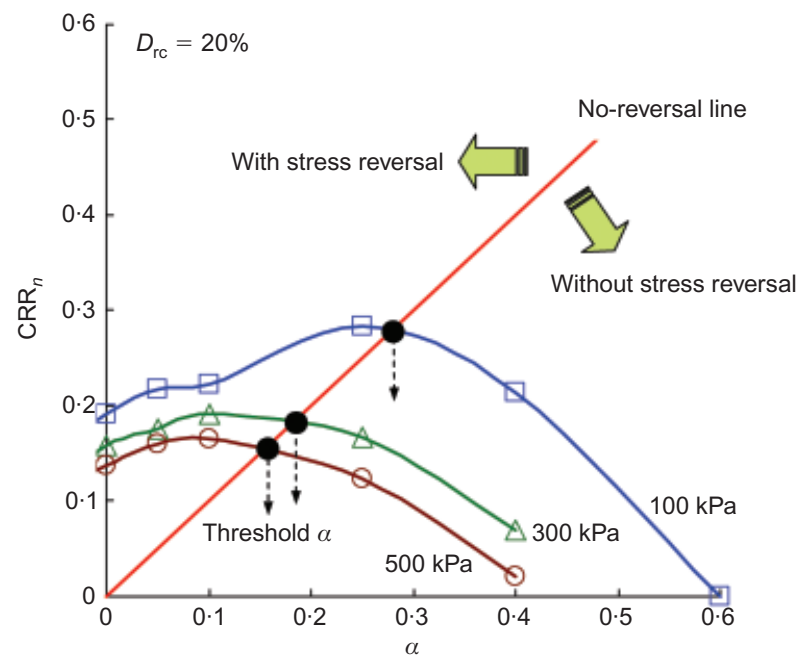

Fig. 18. Determination of threshold $\alpha$ using test data for loose sand

against $\alpha$ will be lower when the sand becomes looser, because of either decreasing $D_{\mathrm{rc}}$ or increasing $\sigma_{\mathrm{nc}}^{\prime}$. This dependence is shown more clearly in Fig. 19, where the threshold $\alpha$ is plotted against the confining pressure $\sigma_{\text {nc }}^{\prime}$ for two cases of relative density. It can be seen that, at the same relative density, the threshold $\alpha$ decreases with increasing $\sigma_{\text {nc }}^{\prime}$, while at the same confining pressure it increases with increasing $D_{\text {rc }}$.

Recognising that the state parameter can better describe the initial state, the threshold $\alpha\left(\alpha_{\text {th }}\right)$ is then re-plotted as a function of the state parameter $\psi$ in Fig. 20. Strikingly, a unique relationship exists between the two, which can be represented by a best-fit line as

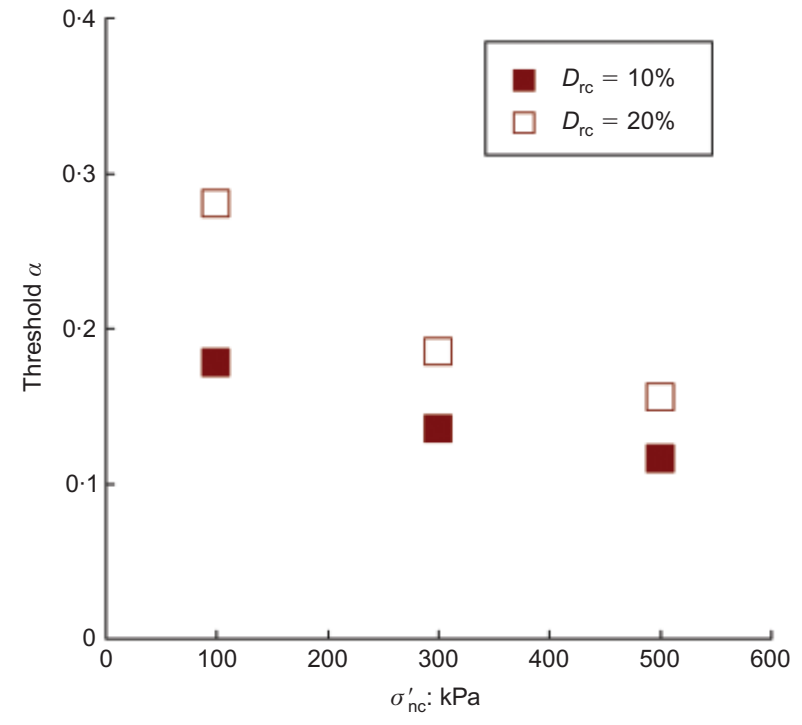

Fig. 19. Dependence of threshold $\alpha$ on confining pressure and relative density

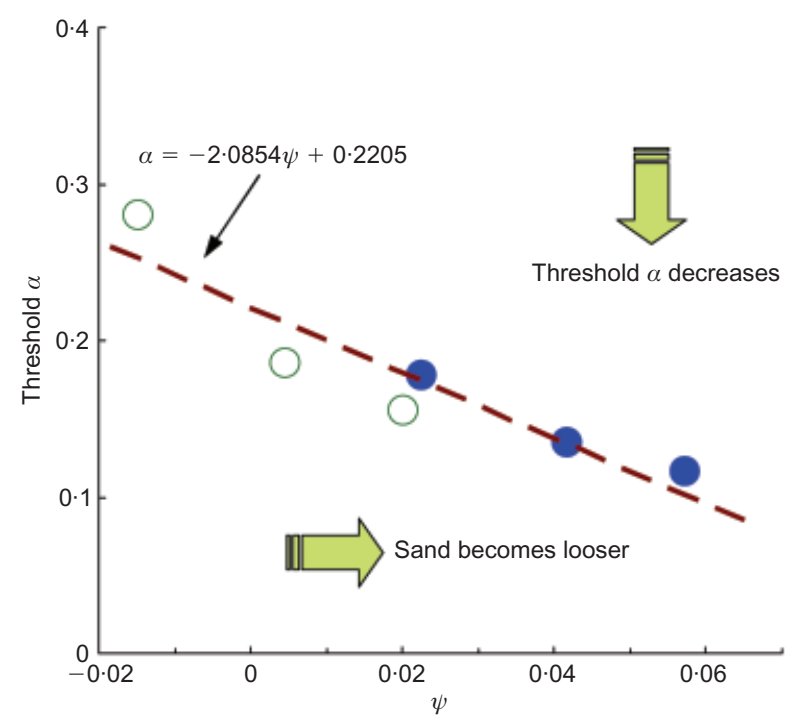

Fig. 20. Threshold $\alpha$ as a function of state parameter

$$
\alpha_{\mathrm{th}}=-2 \cdot 0854 \psi+0.2205
$$

The data points in Fig. 20 are derived from the tests on loose samples at $D_{\mathrm{rc}}=10 \%$ and $20 \%$ only, as these samples show the existence of threshold $\alpha$. An interesting question then arises: Does dense sand have a threshold $\alpha$ ? To answer this question, one may refer back to Fig. 13, where the test data for $D_{\mathrm{rc}}=50 \%$ and $70 \%$ are included along with the data for loose samples. A first glance may suggest that the answer is 'No', at least for the range of $\alpha$ values tested in the study. However, recognising the good correlation shown in Fig. 20, and in line with the CSSM concepts, it is postulated that the concept of threshold $\alpha$ should exist for dense samples at $D_{\mathrm{rc}}=50 \%$ and $70 \%$ as well, if the stress level could become sufficiently high to lead to positive values of $\psi$ (i.e. a loose, contractive state).

The above inference is illustrated in Fig. 21 for the case of dense samples at a relative density of $70 \%$. As can be seen, the position of the $\mathrm{CRR}_{n}$ lines is dependent on the confining pressure level: with increasing $\sigma_{\mathrm{nc}}^{\prime}$ values the $\mathrm{CRR}_{n}$ line tends to be lowered down and, eventually, to cross the no-stress-reversal line. From the intersection of the two lines the threshold $\alpha$ can subsequently be determined. 


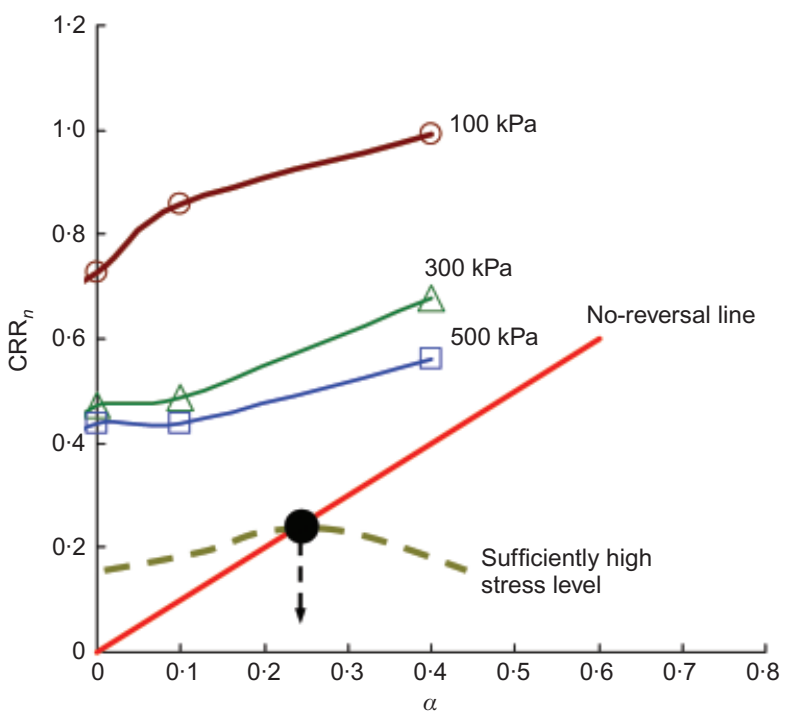

Fig. 21. Inference of existence of threshold $\alpha$ for dense sand samples

Because of the capacity limitation of the triaxial device used, it was not possible to perform tests at confining pressure levels much higher than the current $500 \mathrm{kPa}$ to verify this inference. Nevertheless, by examining the existing data for higher stress levels, it is very encouraging to see evidence in support of the inference. Shown in Fig. 22 are the data interpreted from the triaxial test results of Vaid \& Chern (1985), who performed tests on tailings sand samples with a relative density of $70 \%$ under cell pressures of 200 and $1600 \mathrm{kPa}$. It can be seen that, whereas the sample at $200 \mathrm{kPa}$ showed an increase in cyclic resistance with $\alpha$, the sample at $1600 \mathrm{kPa}$ indeed showed an increase and then a decrease of resistance with $\alpha$. The broken line superimposed on the plot is the no-reversal line introduced previously. Interestingly, it can be seen that the cyclic resistance of the tailings sand is about to reduce when the $\mathrm{CRR}_{n}$ line crosses the no-reversal line: this observation is in good support of the proposed idea (see Figs 18 and 21).

As no information is available for the critical state line of the tailings sand, it is impossible to examine how the data fit in the relationship proposed in Fig. 20, but it is expected

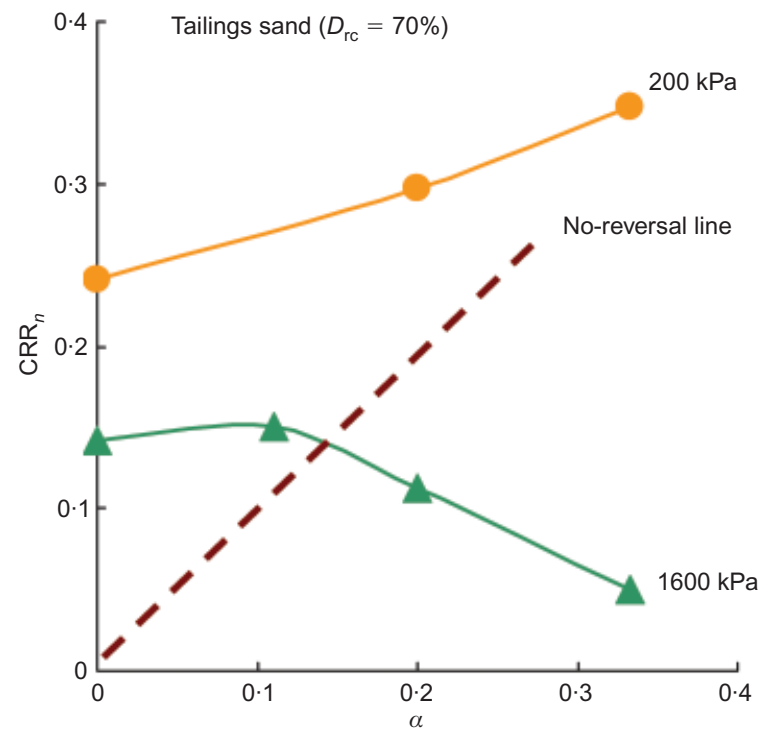

Fig. 22. Supporting evidence for existence of threshold $\alpha$ for dense sand (data interpreted from Vaid \& Chern, 1985) that a trend similar to the proposed one should exist. Obviously, future work on different sands would be of value in validating the idea and interpretations developed here.

\section{SUMMARY AND CONCLUSIONS}

This paper presents a systematic experimental study aimed at investigating the cyclic behaviour of sand under nonsymmetrical loading conditions, and at characterising the role of initial static shear in cyclic liquefaction resistance. An attempt has been made to develop a unified and consistent view of the interrelated effects of various state variables in the framework of CSSM. The main findings resulting from the study can be summarised as follows.

(a) There are three distinctly different failure modes for sand under cyclic triaxial loading conditions. The most critical one is characterised by abrupt, runaway deformations without any warning signal. The second failure mode is that in which cyclic strain in terms of double amplitude accumulates to a large level of deformation. The third type is that in which plastic strain accumulates in one direction to arrive at a specified level of deformation.

(b) The runaway, flow-type failure is pertinent to sufficiently loose sand, and can occur under both stressreversal and non-reversal conditions. Medium dense or dense sand at a normal confining pressure level exhibits either the second or third mode of failure, and stress reversal is a key factor distinguishing them. When no stress reversals occur, the plastic strain accumulation pattern governs.

(c) Under otherwise identical conditions the cyclic resistance of sand always increases with increasing relative density, no matter whether initial static shear stress is present or not, but the effect of relative density appears to depend on the level of static shear stress. At high static shear stress levels $(\alpha=0 \cdot 4)$, the increase of cyclic resistance due to the increase in relative density is more profound than that at low static shear stress levels $(\alpha=0 \cdot 1$ and 0$)$.

(d) Increasing the confining pressure always reduces cyclic resistance, as long as the relative density and the static shear stress ratio remain unchanged. This impact can be crucial for loose sand under high static shear stress levels $(\alpha=0 \cdot 4)$, since the sand may lose its resistance totally when the confining pressure is increased.

(e) For loose sand, the presence of initial static shear stress can be beneficial for or detrimental to its resistance to liquefaction. In this connection, a threshold $\alpha$ exists after which the cyclic resistance of sand tends to reduce with increasing $\alpha$ values. The magnitude of the threshold $\alpha$ is dependent on the initial relative density and the confining pressure.

$(f)$ In the CSSM framework, a fairly good linear relationship exists between the threshold $\alpha$ and the state parameter $\psi$. This relationship indicates that the threshold $\alpha$ decreases with increasing value $\psi$. Along this line, the concept of threshold $\alpha$ can be extended to sand at high relative density, as long as the confining pressure involved is sufficiently high.

\section{ACKNOWLEDGEMENTS}

The work presented in this paper was supported by the Research Grants Council of Hong Kong under grant number 719105. This support is gratefully acknowledged. The support of the University of Hong Kong through its Outstanding 
Young Researcher Award Programme is also highly acknowledged.

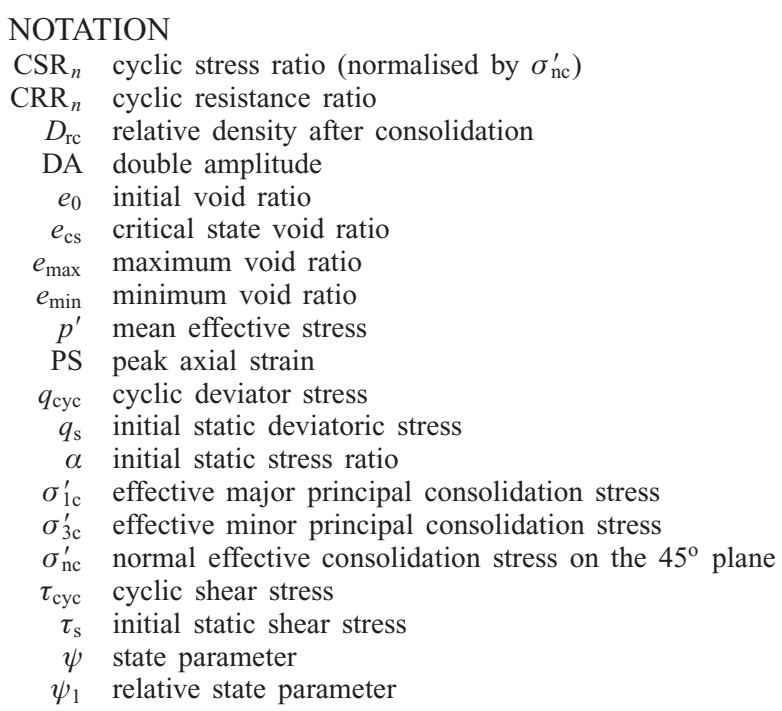

\section{REFERENCES}

Been, K. \& Jefferies, M. (1985). A state parameter for sands. Géotechnique 35, No. 2, 99-112, doi: 10.1680/geot.1985.35. 2.99 .

Been, K. \& Jefferies, M. (2004). Stress-dilatancy in very loose sand. Can. Geotech. J. 41, No. 5, 972-989.

Boulanger, R. W. (2003). Relating $K_{\alpha}$ to relative state parameter index. J. Geotech. Geoenviron. Engng ASCE 129, No. 8, 770773

Castro, G. (1975). Liquefaction and cyclic mobility of saturated sands. J. Geotech. Engng. Div. ASCE 101, No. GT6, 551-569.

Harder, L. F. Jr. \& Boulanger, R. W. (1997). Application of $K_{\sigma}$ and $K_{\alpha}$ correction factors. Proceedings of the NCEER workshop on evaluation of liquefaction resistance of soils, National Center for Earthquake Engineering Research, State University of New York at Buffalo, pp. 167-190.

Hyodo, M., Tanimizu, H., Yasufuku, N. \& Murata, H. (1994). Undrained cyclic and monotonic triaxial behavior of saturated loose sand. Soils Found. 34, No. 1, 19-32.

Ishihara, K. (1993). Liquefaction and flow failure during earthquakes. Géotechnique 43, No. 3, 351-415, doi: 10.1680/ geot.1993.43.3.351.

Ishihara, K. (1996). Soil behaviour in earthquake geotechnics. Oxford: Clarendon Press.

Konrad, J. M. (1988). Interpretation of flat plate dilatometer tests in sands in terms of the state parameter. Géotechnique 38, No. 2, 263-277, doi: 10.1680/geot.1988.38.2.263.

Ladd, R. S. (1974). Specimen preparation and liquefaction of sands. J. Geotech. Engng Div. ASCE 100, No. GT10, 1180-1184.

Lee, K. L. \& Seed, H. B. (1967). Cyclic stress conditions causing liquefaction of sand. J. Soil Mech. Found. Div. ASCE 93, No. SM1, 47-70

Schofield, A. N. \& Wroth, C. P. (1968). Critical state soil mechanics. London: McGraw-Hill.

Seed, H. B. (1983). Earthquake-resistant design of earth dams. Proceedings of the symposium on seismic design of earth dams and caverns, New York, pp. 41-64.

Seed, H. B. \& Lee, K. L. (1966). Liquefaction of saturated sands during cyclic loading. J. Soil Mech. Found. Div. ASCE 92, No. SM6, $105-134$.

Seed, R. B. \& Harder, L. F. Jr. (1990). SPT-based analysis of cyclic pore pressure generation and undrained residual strength. Proceedings of the H. Bolton Seed Memorial Symposium, pp. 351376. Vancouver: BiTech Publishers.

Selig, E. T. \& Chang, C. S. (1981). Soil failure modes in undrained cyclic loading. J. Geotech. Engng Div. ASCE 107, No. GT5, $539-551$.

Sladen, J. A., D'Hollander, R. D. \& Krahn, J. (1985). The liquefaction of sands: a collapse surface approach. Can. Geotech. J. 22, No. 4, 564-578.

Toki, S., Tatsuoka, F., Miura, S., Yoshimi, Y., Yasuda, S. \& Makihara, Y. (1986). Cyclic undrained triaxial strength of sand by a cooperative test program. Soils Found. 26, No. 3, 117-128.

Vaid, Y. P. \& Chern, J. C. (1983). Effect of static shear on resistance to liquefaction. Soils Found. 23, No. 1, 47-60.

Vaid, Y. P. \& Chern, J. C. (1985). Cyclic and monotonic undrained response of sands. In Advances in the art of testing soils under cyclic loading conditions (ed. V. Khosla), pp. 171-176. Reston, VA: ASCE

Vaid, Y. P., Stedman, J. D. \& Sivathayalan, S. (2001). Confining stress and static shear effects in cyclic liquefaction. Can. Geotech. J. 38, No. 3, 580-591.

Verdugo, R. \& Ishihara, K. (1996). The steady state of sandy soils. Soils Found. 36, No. 2, 81-91.

Wood, D. M. (1990). Soil behaviour and critical state soil mechanics. Cambridge: Cambridge University Press.

Wood, D. M., Belkheir, K. \& Liu, D. F. (1994). Strain softening and state parameter for sand modelling. Géotechnique 44, No. 2, 335-339, doi: 10.1680/geot.1994.44.2.335.

Yang, J. (2002a). Liquefaction resistance of sand in relation to P-wave velocity. Géotechnique 52, No. 4, 295-298, doi: 10.1680/geot.2002.52.4.295.

Yang, J. (2002b). Non-uniqueness of flow liquefaction line for loose sand. Géotechnique 52, No. 10, 757-760, doi: 10.1680/ geot.2002.52.10.757.

Yang, J. \& Li, X. S. (2004). State-dependent strength of sands from the perspective of unified modeling. J. Geotech. Geoenviron. Engng ASCE 130, No. 2, 186-198.

Yang, J., Savidis, S. \& Roemer, M. (2004). Evaluating liquefaction strength of partially saturated sand. J. Geotech. Geoenviron. Engng ASCE 130, No. 9, 975-979.

Yoshimi, Y. K. \& Oh-oka, H. (1975). Influence of degree of shear stress reversal on the liquefaction potential of saturated sand. Soils Found. 15, No. 3, 27-40.

Youd, T. L., Idriss, I. M., Andrus, R. D. et al. (2001). Liquefaction resistance of soils: summary report from the 1996 NCEER and 1998 NCEER/NSF workshops on evaluation of liquefaction resistance of soils. J. Geotech. Geoenviron. Engng. ASCE 127, No. $10,817-833$. 